\title{
Measurement of the muon neutrino inclusive charged-current cross section in the energy range of 1-3 GeV with the T2K INGRID detector
}

K. Abe, ${ }^{46}$ C. Andreopoulos, ${ }^{44,26}$ M. Antonova, ${ }^{21}$ S. Aoki, ${ }^{23}$ A. Ariga, ${ }^{1}$ S. Assylbekov, ${ }^{7}$ D. Autiero, ${ }^{28}$ M. Barbi, ${ }^{39}$ G.J. Barker, ${ }^{54}$ G. Barr ${ }^{35}$ P. Bartet-Friburg, ${ }^{36}$ M. Batkiewicz, ${ }^{12}$ F. Bay, ${ }^{10}$ V. Berardi, ${ }^{17}$ S. Berkman, ${ }^{3}$ S. Bhadra, ${ }^{58}$ A. Blondel ${ }^{11}$ S. Bolognesi, ${ }^{5}$ S. Bordoni, ${ }^{14}$ S.B. Boyd,${ }^{54}$ D. Brailsford ${ }^{25,}{ }^{26}$ A. Bravar, ${ }^{11}$ C. Bronner, ${ }^{22}$ R.G. Calland, ${ }^{22}$ S. Cao, ${ }^{24}$ J. Caravaca Rodríguez, ${ }^{14}$ S.L. Cartwright, ${ }^{42}$ R. Castillo, ${ }^{14}$ M.G. Catanesi, ${ }^{17}$ A. Cervera, ${ }^{15}$ D. Cherdack, ${ }^{7}$ N. Chikuma, ${ }^{45}$ G. Christodoulou, ${ }^{26}$ A. Clifton, ${ }^{7}$ J. Coleman, ${ }^{26}$ G. Collazuol, ${ }^{19}$ L. Cremonesi, ${ }^{38}$ A. Dabrowska, ${ }^{12}$ G. De Rosa, ${ }^{18}$ T. Dealtry, ${ }^{25}$ S.R. Dennis, ${ }^{54,44}$ C. Densham, ${ }^{44}$ D. Dewhurst,${ }^{35}$ F. Di Lodovico, ${ }^{38}$ S. Di Luise, ${ }^{10}$ S. Dolan, ${ }^{35}$

O. Drapier, ${ }^{9}$ K. Duffy, ${ }^{35}$ J. Dumarchez,${ }^{36}$ S. Dytman, ${ }^{37}$ M. Dziewiecki, ${ }^{53}$ S. Emery-Schrenk, ${ }^{5}$ A. Ereditato, ${ }^{1}$

T. Feusels, ${ }^{3}$ A.J. Finch, ${ }^{25}$ G.A. Fiorentini, ${ }^{58}$ M. Friend, ${ }^{13}{ }^{, ~ a}$ Y. Fujii, ${ }^{13}{ }^{, ~ a}$ D. Fukuda, ${ }^{33}$ Y. Fukuda, ${ }^{30}$ A.P. Furmanski, ${ }^{54}$ V. Galymov, ${ }^{28}$ A. Garcia, ${ }^{14}$ S. Giffin, ${ }^{39}$ C. Giganti, ${ }^{36}$ K. Gilje, ${ }^{32}$ M. Gonin, ${ }^{9}$ N. Grant,${ }^{25}$ D.R. Hadley,${ }^{54}$ L. Haegel,${ }^{11}$ M.D. Haigh, ${ }^{54}$ P. Hamilton, ${ }^{16}$ D. Hansen, ${ }^{37}$ T. Hara, ${ }^{23}$ M. Hartz $,{ }^{22,}, 50$ T. Hasegawa, ${ }^{13}$ a N.C. Hastings ${ }^{39}$ T. Hayashino, ${ }^{24}$ Y. Hayato, ${ }^{46,}{ }^{22}$ R.L. Helmer, ${ }^{50}$ M. Hierholzer, ${ }^{1}$ A. Hillairet,${ }^{51}$ A. Himmel, ${ }^{8}$ T. Hiraki,${ }^{24}$ S. Hirota, ${ }^{24}$ J. Holeczek, ${ }^{43}$ S. Horikawa, ${ }^{10}$ F. Hosomi ${ }^{45}$ K. Huang, ${ }^{24}$ A.K. Ichikawa, ${ }^{24}$ K. Ieki, ${ }^{24}$ M. Ikeda, ${ }^{46}$ J. Imber, ${ }^{9}$ J. Insler,${ }^{27}$

R.A. Intonti, ${ }^{17}$ T.J. Irvine, ${ }^{47}$ T. Ishida, ${ }^{13}$ a T. Ishii,,${ }^{13,}$ a E. Iwai, ${ }^{13}$ K. Iwamoto, ${ }^{40}$ A. Izmaylov, ${ }^{15,21}$ A. Jacob, ${ }^{35}$ B. Jamieson, ${ }^{56}$ M. Jiang, ${ }^{24}$ S. Johnson, ${ }^{6}$ J.H. Jo ${ }^{32}$ P. Jonsson, ${ }^{16}$ C.K. Jung, ${ }^{32}$, b M. Kabirnezhad, ${ }^{31}$ A.C. Kaboth, ${ }^{16}$ T. Kajita ${ }^{47,}$ b H. Kakuno, ${ }^{48}$ J. Kameda, ${ }^{46}$ D. Karlen, ${ }^{51,}{ }^{50}$ I. Karpikov, ${ }^{21}$ T. Katori, ${ }^{38}$ E. Kearns, ${ }^{2,}$ 22, b

M. Khabibullin, ${ }^{21}$ A. Khotjantsev, ${ }^{21}$ D. Kielczewska, ${ }^{52}$ T. Kikawa, ${ }^{24}$ H. Kim,${ }^{34}$ J. Kim, ${ }^{3}$ S. King,${ }^{38}$ J. Kisiel, ${ }^{43}$ T. Kobayashi, ${ }^{13, \text { a }}$ L. Koch,,${ }^{41}$ T. Koga ${ }^{45}$ A. Konaka, ${ }^{50}$ K. Kondo, ${ }^{24}$ A. Kopylov,${ }^{21}$ L.L. Kormos, ${ }^{25}$ A. Korzenev, ${ }^{11}$

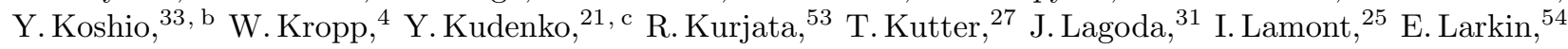
M. Laveder, ${ }^{19}$ M. Lawe, ${ }^{25}$ M. Lazos,${ }^{26}$ T. Lindner,${ }^{50}$ Z.J. Liptak,${ }^{6}$ R.P. Litchfield,${ }^{54}$ A. Longhin, ${ }^{19}$ J.P. Lopez, ${ }^{6}$ L. Ludovici, ${ }^{20}$ X. Lu ${ }^{35}$ L. Magaletti, ${ }^{17}$ K. Mahn,${ }^{29}$ M. Malek ${ }^{42}$ S. Manly,${ }^{40}$ A.D. Marino, ${ }^{6}$ J. Marteau, ${ }^{28}$ J.F. Martin, ${ }^{49}$ P. Martins, ${ }^{38}$ S. Martynenko, ${ }^{32}$ T. Maruyama, ${ }^{13}{ }^{\text {a a }}$ V. Matveev,${ }^{21}$ K. Mavrokoridis, ${ }^{26}$ W.Y. Ma, ${ }^{16}$ E. Mazzucato, ${ }^{5}$ M. McCarthy, ${ }^{58}$ N. McCauley, ${ }^{26}$ K.S. McFarland, ${ }^{40}$ C. McGrew, ${ }^{32}$ A. Mefodiev, ${ }^{21}$ M. Mezzetto, ${ }^{19}$ P. Mijakowski, ${ }^{31}$ C.A. Miller, ${ }^{50}$ A. Minamino, ${ }^{24}$ O. Mineev ${ }^{21}$ S. Mine,${ }^{4}$ A. Missert,${ }^{6}$ M. Miura ${ }^{46, b}$ S. Moriyama, ${ }^{46, b}$ Th.A. Mueller ${ }^{9}$

S. Murphy, ${ }^{10}$ J. Myslik, ${ }^{51}$ T. Nakadaira, ${ }^{13,}$ a M. Nakahata, ${ }^{46,22}$ K.G. Nakamura, ${ }^{24}$ K. Nakamura, ${ }^{22,13, \text { a }}$

K.D. Nakamura, ${ }^{24}$ S. Nakayama, ${ }^{46,}$ b T. Nakaya, ${ }^{24,}{ }^{22}$ K. Nakayoshi, ${ }^{13}$, a C. Nantais, ${ }^{3}$ C. Nielsen, ${ }^{3}$ M. Nirkko, ${ }^{1}$ K. Nishikawa, ${ }^{13, \text { a }}$ Y. Nishimura, ${ }^{47}$ J. Nowak, ${ }^{25}$ H.M. O'Keeffe, ${ }^{25}$ R. Ohta, ${ }^{13}{ }^{\text {, a }}$ K. Okumura, ${ }^{47,}{ }^{22}$ T. Okusawa, ${ }^{34}$ W. Oryszczak, ${ }^{52}$ S.M. Oser ${ }^{3}$ T. Ovsyannikova, ${ }^{21}$ R.A. Owen,${ }^{38}$ Y. Oyama, ${ }^{13}$, a V. Palladino, ${ }^{18}$ J.L. Palomino, ${ }^{32}$ V. Paolone ${ }^{37}$ D. Payne, ${ }^{26}$ J.D. Perkin, ${ }^{42}$ Y. Petrov ${ }^{3}$ L. Pickard, ${ }^{42}$ L. Pickering, ${ }^{16}$ E.S. Pinzon Guerra ${ }^{58}$ C. Pistillo, ${ }^{1}$ B. Popov, ${ }^{36, d}$ M. Posiadala-Zezula, ${ }^{52}$ J.-M. Poutissou, ${ }^{50}$ R. Poutissou, ${ }^{50}$ P. Przewlocki, ${ }^{31}$ B. Quilain, ${ }^{24}$ E. Radicioni, ${ }^{17}$

P.N. Ratoff, ${ }^{25}$ M. Ravonel, ${ }^{11}$ M.A.M. Rayner ${ }^{11}$ A. Redij, ${ }^{1}$ E. Reinherz-Aronis, ${ }^{7}$ C. Riccio, ${ }^{18}$ P. Rojas, ${ }^{7}$ E. Rondio,${ }^{31}$

S. Roth, ${ }^{41}$ A. Rubbia, ${ }^{10}$ A. Rychter, ${ }^{53}$ R. Sacco ${ }^{38}$ K. Sakashita, ${ }^{13,}{ }^{2}$ F. Sánchez, ${ }^{14}$ F. Sato, ${ }^{13}$ E. Scantamburlo, ${ }^{11}$

K. Scholberg, ${ }^{8, ~ b}$ S. Schoppmann, ${ }^{41}$ J.D. Schwehr,${ }^{7}$ M. Scott, ${ }^{50}$ Y. Seiya, ${ }^{34}$ T. Sekiguchi, ${ }^{13,}$, a H. Sekiya, ${ }^{46,22, ~ b}$

D. Sgalaberna, ${ }^{10}$ R. Shah, ${ }^{44,35}$ A. Shaikhiev, ${ }^{21}$ F. Shaker, ${ }^{56}$ D. Shaw,${ }^{25}$ M. Shiozawa, ${ }^{46,}{ }^{22}$ T. Shirahige, ${ }^{33}$

S. Short, ${ }^{38}$ M. Smy ${ }^{4}$ J.T. Sobczyk,${ }^{57}$ M. Sorel,${ }^{15}$ L. Southwell, ${ }^{25}$ P. Stamoulis, ${ }^{15}$ J. Steinmann ${ }^{41}$ T. Stewart ${ }^{44}$ Y.Suda, ${ }^{45}$ S. Suvorov, ${ }^{21}$ A.Suzuki, ${ }^{23}$ K. Suzuki, ${ }^{24}$ S.Y.Suzuki, ${ }^{13,}$ a Y. Suzuki, ${ }^{22,}{ }^{22}$ R. Tacik,${ }^{39,}{ }^{50}$ M. Tada, ${ }^{13}$, a S. Takahashi, ${ }^{24}$ A. Takeda, ${ }^{46}$ Y. Takeuchi, ${ }^{23,}{ }^{22}$ H.K. Tanaka, ${ }^{46, \text { b }}$ H.A. Tanaka, ${ }^{49,}{ }^{50,}{ }^{\text {e }}$ D. Terhorst,${ }^{41}$ R. Terri, ${ }^{38}$

L.F. Thompson, ${ }^{42}$ S. Tobayama, ${ }^{3}$ W. Toki,${ }^{7}$ T. Tomura,${ }^{46}$ C. Touramanis,${ }^{26}$ T. Tsukamoto,,${ }^{13, \text { a }}$ M. Tzanov ${ }^{27}$ Y. Uchida, ${ }^{16}$ A. Vacheret, ${ }^{35}$ M. Vagins, ${ }^{22,4}$ Z. Vallari, ${ }^{32}$ G. Vasseur, ${ }^{5}$ T. Wachala,,${ }^{12}$ K. Wakamatsu, ${ }^{34}$ C.W. Walter, ${ }^{8, ~ b}$ D. Wark ${ }^{44,35}$ W. Warzycha, ${ }^{52}$ M.O. Wascko, ${ }^{16}$ A. Weber, ${ }^{44,}{ }^{35}$ R. Wendell, ${ }^{46}$, b R.J. Wilkes,${ }^{55}$ M.J. Wilking, ${ }^{32}$ C. Wilkinson, ${ }^{1}$ J.R. Wilson, ${ }^{38}$ R.J. Wilson, ${ }^{7}$ Y. Yamada, ${ }^{13,}{ }^{a}$ K. Yamamoto,${ }^{34}$ M. Yamamoto, ${ }^{24}$ C. Yanagisawa, ${ }^{32, f}$ T. Yano, ${ }^{23}$ S. Yen, ${ }^{50}$ N. Yershov, ${ }^{21}$ M. Yokoyama, ${ }^{45, ~ b}$ J. Yoo, ${ }^{27}$ K. Yoshida, ${ }^{24}$ T. Yuan, ${ }^{6}$ M. Yu, ${ }^{58}$ A. Zalewska ${ }^{12}$

J. Zalipska, ${ }^{31}$ L. Zambelli, ${ }^{13}$ a K. Zaremba, ${ }^{53}$ M.Ziembicki, ${ }^{53}$ E.D. Zimmerman, ${ }^{6}$ M. Zito, ${ }^{5}$ and J. Żmuda ${ }^{57}$

(The T2K Collaboration)

\footnotetext{
${ }^{1}$ University of Bern, Albert Einstein Center for Fundamental Physics, Laboratory for High Energy Physics (LHEP), Bern, Switzerland

${ }^{2}$ Boston University, Department of Physics, Boston, Massachusetts, U.S.A.

${ }^{3}$ University of British Columbia, Department of Physics and Astronomy, Vancouver, British Columbia, Canada

${ }^{4}$ University of California, Irvine, Department of Physics and Astronomy, Irvine, California, U.S.A.

${ }^{5}$ IRFU, CEA Saclay, Gif-sur-Yvette, France

${ }^{6}$ University of Colorado at Boulder, Department of Physics, Boulder, Colorado, U.S.A.

${ }^{7}$ Colorado State University, Department of Physics, Fort Collins, Colorado, U.S.A.
} 
${ }^{8}$ Duke University, Department of Physics, Durham, North Carolina, U.S.A.

${ }^{9}$ Ecole Polytechnique, IN2P3-CNRS, Laboratoire Leprince-Ringuet, Palaiseau, France

${ }^{10}$ ETH Zurich, Institute for Particle Physics, Zurich, Switzerland

${ }^{11}$ University of Geneva, Section de Physique, DPNC, Geneva, Switzerland

${ }^{12}$ H. Niewodniczanski Institute of Nuclear Physics PAN, Cracow, Poland

${ }^{13}$ High Energy Accelerator Research Organization (KEK), Tsukuba, Ibaraki, Japan

${ }^{14}$ Institut de Fisica d'Altes Energies (IFAE), The Barcelona Institute of Science and Technology, Campus UAB, Bellaterra (Barcelona) Spain

${ }^{15}$ IFIC (CSIC \& University of Valencia), Valencia, Spain

${ }^{16}$ Imperial College London, Department of Physics, London, United Kingdom

${ }^{17}$ INFN Sezione di Bari and Università e Politecnico di Bari, Dipartimento Interuniversitario di Fisica, Bari, Italy

${ }^{18}$ INFN Sezione di Napoli and Università di Napoli, Dipartimento di Fisica, Napoli, Italy

${ }^{19}$ INFN Sezione di Padova and Università di Padova, Dipartimento di Fisica, Padova, Italy

${ }^{20}$ INFN Sezione di Roma and Università di Roma "La Sapienza", Roma, Italy

${ }^{21}$ Institute for Nuclear Research of the Russian Academy of Sciences, Moscow, Russia

${ }^{22}$ Kavli Institute for the Physics and Mathematics of the Universe (WPI), The University

of Tokyo Institutes for Advanced Study, University of Tokyo, Kashiwa, Chiba, Japan

${ }^{23}$ Kobe University, Kobe, Japan

${ }^{24}$ Kyoto University, Department of Physics, Kyoto, Japan

${ }^{25}$ Lancaster University, Physics Department, Lancaster, United Kingdom

${ }^{26}$ University of Liverpool, Department of Physics, Liverpool, United Kingdom

${ }^{27}$ Louisiana State University, Department of Physics and Astronomy, Baton Rouge, Louisiana, U.S.A.

${ }^{28}$ Université de Lyon, Université Claude Bernard Lyon 1, IPN Lyon (IN2P3), Villeurbanne, France

${ }^{29}$ Michigan State University, Department of Physics and Astronomy, East Lansing, Michigan, U.S.A.

${ }^{30}$ Miyagi University of Education, Department of Physics, Sendai, Japan

${ }^{31}$ National Centre for Nuclear Research, Warsaw, Poland

${ }^{32}$ State University of New York at Stony Brook, Department of Physics and Astronomy, Stony Brook, New York, U.S.A.

${ }^{33}$ Okayama University, Department of Physics, Okayama, Japan

${ }^{34}$ Osaka City University, Department of Physics, Osaka, Japan

${ }^{35}$ Oxford University, Department of Physics, Oxford, United Kingdom

${ }^{36}$ UPMC, Université Paris Diderot, CNRS/IN2P3, Laboratoire de

Physique Nucléaire et de Hautes Energies (LPNHE), Paris, France

${ }^{37}$ University of Pittsburgh, Department of Physics and Astronomy, Pittsburgh, Pennsylvania, U.S.A.

${ }^{38}$ Queen Mary University of London, School of Physics and Astronomy, London, United Kingdom

${ }^{39}$ University of Regina, Department of Physics, Regina, Saskatchewan, Canada

${ }^{40}$ University of Rochester, Department of Physics and Astronomy, Rochester, New York, U.S.A.

${ }^{41}$ RWTH Aachen University, III. Physikalisches Institut, Aachen, Germany

${ }^{42}$ University of Sheffield, Department of Physics and Astronomy, Sheffield, United Kingdom

${ }^{43}$ University of Silesia, Institute of Physics, Katowice, Poland

${ }^{44}$ STFC, Rutherford Appleton Laboratory, Harwell Oxford, and Daresbury Laboratory, Warrington, United Kingdom

${ }^{45}$ University of Tokyo, Department of Physics, Tokyo, Japan

${ }^{46}$ University of Tokyo, Institute for Cosmic Ray Research, Kamioka Observatory, Kamioka, Japan

${ }^{47}$ University of Tokyo, Institute for Cosmic Ray Research, Research Center for Cosmic Neutrinos, Kashiwa, Japan

${ }^{48}$ Tokyo Metropolitan University, Department of Physics, Tokyo, Japan

${ }^{49}$ University of Toronto, Department of Physics, Toronto, Ontario, Canada

${ }^{50}$ TRIUMF, Vancouver, British Columbia, Canada

${ }^{51}$ University of Victoria, Department of Physics and Astronomy, Victoria, British Columbia, Canada

${ }^{52}$ University of Warsaw, Faculty of Physics, Warsaw, Poland

${ }^{53}$ Warsaw University of Technology, Institute of Radioelectronics, Warsaw, Poland

${ }^{54}$ University of Warwick, Department of Physics, Coventry, United Kingdom

${ }^{55}$ University of Washington, Department of Physics, Seattle, Washington, U.S.A.

${ }^{56}$ University of Winnipeg, Department of Physics, Winnipeg, Manitoba, Canada

${ }^{57}$ Wroclaw University, Faculty of Physics and Astronomy, Wroclaw, Poland

${ }^{58}$ York University, Department of Physics and Astronomy, Toronto, Ontario, Canada

(Dated: September 24, 2015)

We report a measurement of the $\nu_{\mu}$-nucleus inclusive charged current cross section $\left(=\sigma^{c c}\right)$ on iron using data from exposed to the J-PARC neutrino beam. The detector consists of 14 modules in total, which are spread over a range of off-axis angles from $0^{\circ}$ to $1.1^{\circ}$. The variation in the neutrino energy spectrum as a function of the off-axis angle, combined with event topology information, is used to calculate this cross section as a function of neutrino energy. The cross section is measured to be $\sigma^{c c}(1.1 \mathrm{GeV})=1.10 \pm 0.15\left(10^{-38} \mathrm{~cm}^{2} /\right.$ nucleon $), \sigma^{c c}(2.0 \mathrm{GeV})=2.07 \pm 0.27\left(10^{-38} \mathrm{~cm}^{2} /\right.$ nucleon $)$, and $\sigma^{c c}(3.3 \mathrm{GeV})=2.29 \pm 0.45\left(10^{-38} \mathrm{~cm}^{2} /\right.$ nucleon $)$, at energies of $1.1,2.0$, and $3.3 \mathrm{GeV}$, respectively. These results are consistent with the cross section calculated by the neutrino interaction 
generators currently used by T2K. More importantly, the method described here opens up a new way to determine the energy dependence of neutrino-nucleus cross sections.

\section{INTRODUCTION}

Many recent long baseline neutrino oscillation experiments use muon neutrino beams, with neutrino energies ranging from sub-GeV to a few $\mathrm{GeV}$. The observed neutrino-nucleus charged-current (CC) interactions are then used to infer neutrino oscillation parameters. In this energy region, CC quasi-elastic and CC single-pion production reactions dominate the total cross section, and so understanding these channels is essential for precision measurements of the oscillation parameters. Measurements of exclusive cross sections, however, are complicated by re-interactions of the final state hadrons as they exit the nucleus, known as final state interactions (FSI). FSI can absorb or produce particles, resulting in a different set of particles entering the detector than would be expected from the initial interaction. For example, the pion from a CC single-pion interaction might be absorbed in the nucleus, so the observable final state is similar to that from a CC quasi-elastic event. The CC inclusive channel is much less sensitive to these effects, since it only requires the detection of a charged lepton (muon) from the interaction. A precise measurement of this channel, combined with exclusive measurements, will help improve our understanding of neutrino interactions in this energy region.

So far, the MINOS and T2K experiments have measured the CC inclusive $\nu_{\mu}$ cross section on iron $[1,2]$ using neutrino beams which cover the few- $\mathrm{GeV}$ region. The former experiment measured the CC inclusive cross section for neutrinos with energies above $3.5 \mathrm{GeV}$ using the MINOS near detector. The latter used the T2K near detector, INGRID, to measure a flux-averaged CC inclusive cross section at a mean energy of $1.51 \mathrm{GeV}$, where the r.m.s spread of the neutrino energy was $0.76 \mathrm{GeV}(0.84$ $\mathrm{GeV}$ ) below (above) this mean energy. The MINER $\nu \mathrm{A}$ experiment also measured the CC inclusive cross section on iron, but only the cross-section ratio of iron to $\mathrm{CH}$ has been published [3]. The CC inclusive cross section on iron has not yet been measured in the $2-3 \mathrm{GeV}$ energy range, and a measurement covering $1-3 \mathrm{GeV}$ would provide a consistency check between the T2K and MINOS results.

The T2K experiment is a long baseline neutrino oscillation experiment in Japan [4]. T2K utilizes an almost

\footnotetext{
${ }^{\text {a }}$ also at J-PARC, Tokai, Japan

$\mathrm{b}$ affiliated member at Kavli IPMU (WPI), the University of Tokyo, Japan

c also at National Research Nuclear University "MEPhI" and Moscow Institute of Physics and Technology, Moscow, Russia

$\mathrm{d}$ also at JINR, Dubna, Russia

e also at Institute of Particle Physics, Canada

$\mathrm{f}$ also at BMCC/CUNY, Science Department, New York, New York, U.S.A.
}

pure $\nu_{\mu}$ beam produced as the decay product of $\pi^{+}$'s and $K^{+}$'s. The beam is first measured by the near detector, ND280, located $280 \mathrm{~m}$ downstream from the pion production target. After traveling $295 \mathrm{~km}$, the neutrinos are then observed at the far detector, Super-Kamiokande. Oscillation parameters are determined by comparing the neutrino interactions observed at the near and far detectors.

T2K was built around the "off-axis beam method", where detectors are intentionally placed off the central axis of the neutrino beam (hereafter beam-axis). The angle with respect to the beam-axis is called the off-axis angle: $\theta_{\mathrm{OA}}$. The direction of the neutrino parent particles is distributed around the beam-axis, so $\theta_{\mathrm{OA}}$ is approximately equal to the angle between the parent particle and neutrino directions. In this case, the energy of a neutrino produced from the two-body decay $\left(\pi \rightarrow \nu_{\mu}+\mu\right)$ can be expressed as follows:

$$
E_{\nu}=\frac{m_{\pi}^{2}-m_{\mu}^{2}}{2\left(E_{\pi}-p_{\pi} \cos \theta_{\mathrm{OA}}\right)},
$$

where $m_{\pi}$ and $m_{\mu}$ are the masses of the pion and muon whilst $E_{\pi}$ and $p_{\pi}$ are the energy and momentum of the pion. The relation between $E_{\nu}$ and $p_{\pi}$ for different $\theta_{\mathrm{OA}} \mathrm{S}$ is plotted in Fig. 1, showing the maximum neutrino energy reducing as $\theta_{\mathrm{OA}}$ increases. This indicates that the energy spectrum of the neutrino beam peaks at a lower energy and has a narrower width as $\theta_{\mathrm{OA}}$ increases. In the T2K experiment, $\theta_{\mathrm{OA}}=2.5^{\circ}$ was chosen so that the neutrino flux peaks around $0.6 \mathrm{GeV}$, an energy which maximizes the oscillation probability of the muon neutrino at the far detector.

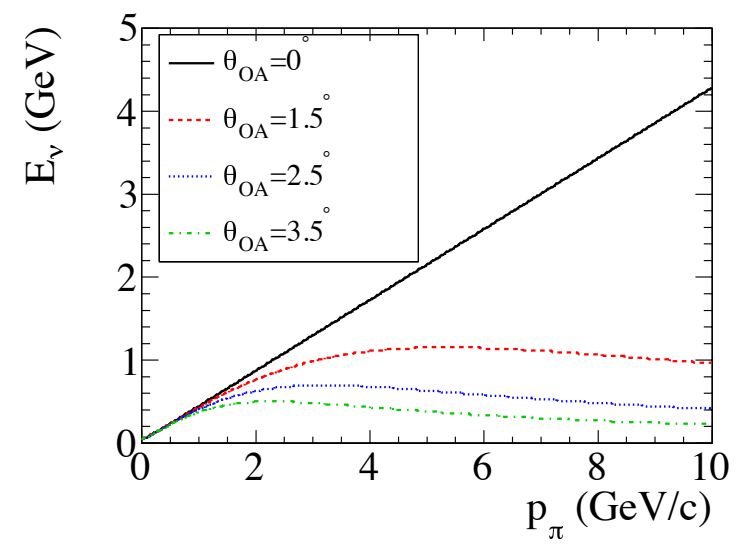

FIG. 1. Neutrino energy as a function of the pion momentum for different $\theta_{\mathrm{OA}} \mathrm{s}$.

The T2K INGRID detector is installed on the beamaxis at the near site. It consists of 14 identical modules, which are spread over a range of $\theta_{\mathrm{OA}}$ from $0^{\circ}$ to $1.1^{\circ}$. Thus, the peak of the neutrino energy spectrum differs among the modules as in Eq. (1). 
In this paper we present a measurement of the $\nu_{\mu}$ inclusive CC cross section on iron in the energy range of 1-3 GeV with INGRID. This analysis uses data collected from 2010 to 2013 , corresponding to $6.27 \times 10^{20}$ protons on target (p.o.t.). The neutrino interactions at different INGRID modules, which are distributed at different positions and thus observed different beam spectra, is used to extract the energy dependence of the cross section. The topology of each event, which is based on the kinematics of the outgoing muon, is also used to further improve the sensitivity of this measurement to the neutrino energy, since the two are directly related. The different energy spectra and event topologies are combined to construct a probability density function (PDF), which is used to measure the cross section using the least $\chi^{2}$ method.

The paper is organized as follows. In Sec. II, we describe the T2K near detector, INGRID. Section III introduces the Monte Carlo (MC) simulation used to predict neutrino event rates at the INGRID detector and describes the systematic uncertainties associated with this model. Next, the analysis method used to extract the energy dependence of the cross section is explained in Sec. IV with a discussion of the remaining systematics in Sec. V. Finally, the result of the analysis is presented in Sec. VI.

\section{THE T2K NEAR DETECTOR: INGRID}

Situated $280 \mathrm{~m}$ downstream from the pion production target, the INGRID detector monitors the neutrino beam direction and intensity. It consists of 14 identical modules, each of which is composed of 9 iron target plates and 11 scintillator tracking planes. The iron plates and the tracking planes are stacked in alternating layers forming a sandwich structure, as shown in Fig. 2.

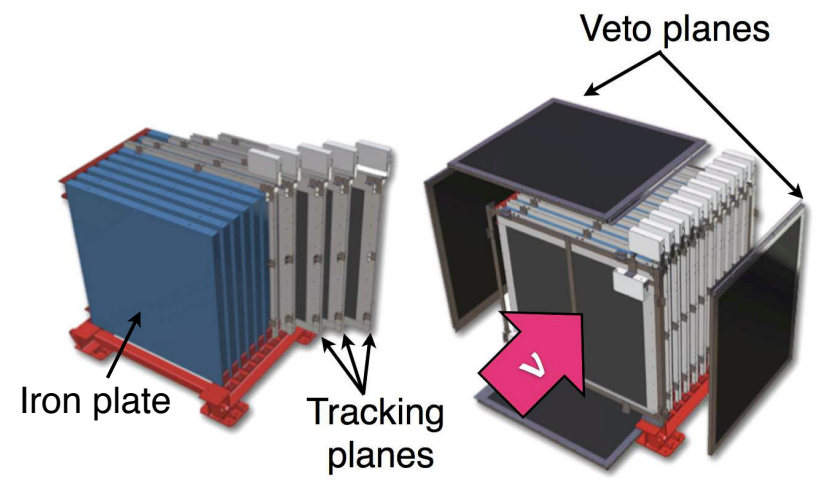

FIG. 2. Schematic drawing of an INGRID module. Each module has 9 iron target plates and 11 tracking planes, with 4 veto planes covering the side surfaces.

Each of the iron plates has dimensions of $124 \times 124 \mathrm{~cm}^{2}$ and a thickness of $6.5 \mathrm{~cm}$, providing a total iron mass of 7.1 ton per module. The module is surrounded by scintillator veto planes, which detect charged particles coming from outside of the module. Each tracking plane has two layers of scintillator bars aligned orthogonally to one another, enabling particles to be tracked in all 3 dimensions as they pass through the plane. The veto planes are also formed from scintillator bars. The bars are coated in $\mathrm{TiO}_{2}$ reflectors to help contain scintillation light, which is then captured by wavelength shifting (WLS) fibers which run through the center of the bars. This light is then read out by a Multi-Pixel Photon Counter (MPPC) [5, 6] and the resultant signal is digitized by the Trip-t front-end board (TFB) [7], to give the integrated charge and timing information. The integration cycle of the electronics is synchronized with the neutrino beam pulse structure, ensuring all data is captured.

The modules are installed in a cross shape centered on the beam-axis. An overview of the INGRID detector is shown on the top in Fig. 3. An ID is assigned to each module as shown on the bottom in Fig 3. Further details of the detector and the basic performance of INGRID can be found in Ref. [8].

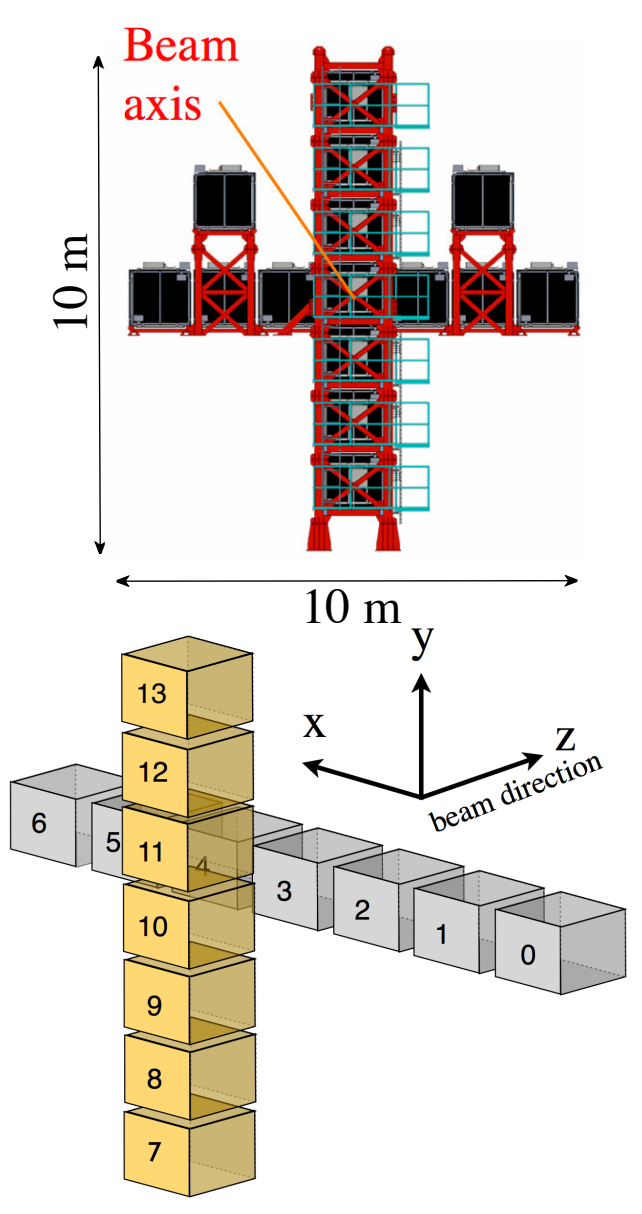

FIG. 3. Top: The INGRID detector. The modules are arranged in a $10 \mathrm{~m}$ x $10 \mathrm{~m}$ cross. The two off-axis modules not located in the arms of the cross are not used in this analysis. Bottom: Module ID given to each module. 


\section{SIMULATING NEUTRINO INTERACTIONS IN THE INGRID DETECTOR}

In this analysis the cross section is measured by comparing data to a prediction, which is calculated using three sequential MC simulations:

1. Prediction of the neutrino flux at the INGRID modules.

2. Simulation of the neutrino-nucleus interactions in the iron target $\left({ }^{56} \mathrm{Fe}\right)$.

3. Propagation of final state particles through the detector and modeling of its response.

After these processes, an event selection, which is detailed in Sec. IV B, is applied to the output in the same way as the data.

\section{A. Neutrino flux \\ 1. Flux prediction}

A detailed description of the neutrino flux prediction can be found in Ref. [9]. In the simulation, protons are impinged upon the carbon target to produce hadrons, which decay into neutrinos. FLUKA2008 [10] and GEANT/GCALOR [11] are used to model hadron production in the target and surrounding material. Propagation of the resulting particles through the electromagnetic horns, which focus the charged hadrons along the beam-axis, is simulated using dedicated, GEANT3 [12]based code, which also models the subsequent decay of the particles. For each hadron decay mode which produces neutrinos, the probability of the neutrinos to be emitted in the direction of the INGRID detector is calculated. The flux prediction is obtained by weighting the generated neutrinos with these probabilities. The flux is then tuned using hadron interaction data, primarily from the NA61/SHINE experiment [13]. Other hadron production data (Eichten et al. [14] and Allaby et al. [15]) are also used to tune the simulation in regions of the hadron production phase space that are not covered by the current NA61/SHINE measurement. In this analysis, the NA61/SHINE data taken in 2007 is used to correct the neutrino flux $[16,17]$.

The neutrino flavor content across different energy regions at module 3 (one of the center modules) is summarized in Table I. This shows that muon neutrinos account for $>\sim 95 \%$ of the total flux for $E_{\nu}<3 \mathrm{GeV}$. The muon neutrino flux fraction then falls to less than $90 \%$ for $E_{\nu}>3 \mathrm{GeV}$, where $\bar{\nu}_{\mu}+\nu_{e}$ account for $>\sim 10 \%$ of the total flux. The flavor content of the neutrino flux at the other modules is similar to that at module 3 .

Figure 4 shows the obtained muon neutrino flux spectra at the INGRID modules. The neutrino energy spectrum changes with module position, with the spectrum
TABLE I. Fraction of the integrated flux by neutrino flavor in each energy range at module 3 .

\begin{tabular}{lrrrrr}
\hline \hline & \multicolumn{5}{c}{ Neutrino energy range $(\mathrm{GeV})$} \\
Flavor & $0-1$ & $1-2$ & $2-3$ & $3-4$ & $>4$ \\
\hline$\nu_{\mu}$ & $94.2 \%$ & $96.8 \%$ & $95.4 \%$ & $89.7 \%$ & $86.5 \%$ \\
$\bar{\nu}_{\mu}$ & $4.8 \%$ & $2.7 \%$ & $3.8 \%$ & $7.9 \%$ & $9.3 \%$ \\
$\nu_{e}$ & $0.9 \%$ & $0.5 \%$ & $0.7 \%$ & $2.0 \%$ & $3.5 \%$ \\
$\bar{\nu}_{e}$ & $0.1 \%$ & $0.0 \%$ & $0.1 \%$ & $0.3 \%$ & $0.6 \%$ \\
\hline \hline
\end{tabular}

at module 0 softer than that at module 3 . This is because module 0 is located at $\theta_{O A}=1.2^{\circ}$ from the neutrino beam-axis, and so the neutrino flux passing through it is shifted to lower energies due to the off-axis beam effect. This feature is, indeed, essential for this crosssection measurement.

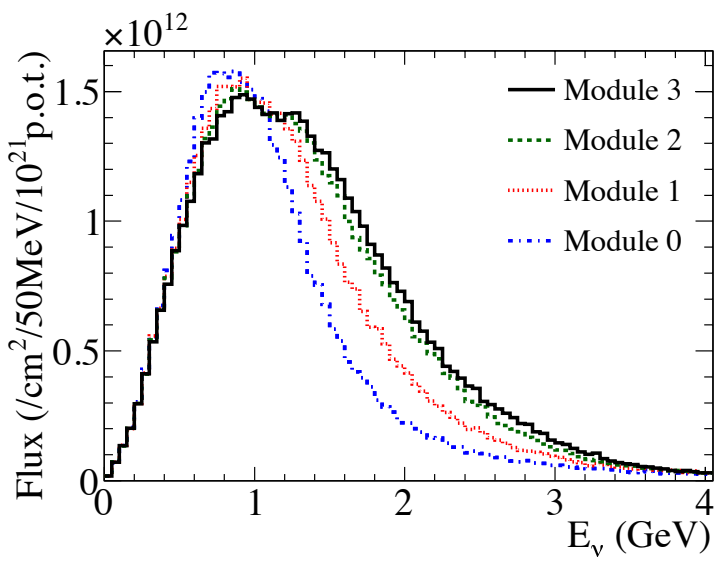

FIG. 4. Muon neutrino fluxes at modules $0,1,2$ and 3.

\section{Flux uncertainties}

The systematic error on the neutrino flux prediction comes from uncertainties in hadron production and from errors in the measurement of the proton beam, the horn current, and the target alignment. The uncertainties in the hadron production are mainly driven by uncertainties in the NA61/SHINE measurements.and those in measurements by Eichten et al. and Allaby et al.. The second category of flux errors is associated with inherent uncertainties and operational variations in the beamline conditions. They include uncertainties in the proton beam position, the beam direction, the absolute horn current, the horn angular alignment, the horn field asymmetry, the target alignment and the proton beam intensity. The method used to estimate these flux uncertainties is described in Ref. [9]. Figure 5 shows the flux error at module 3, which includes all sources of uncertainty, and demonstrates that the systematic error on the neutrino flux is dominated by the uncertainties in the hadron production model. The propagation of the flux error in this analysis is described in Sec. V A. 


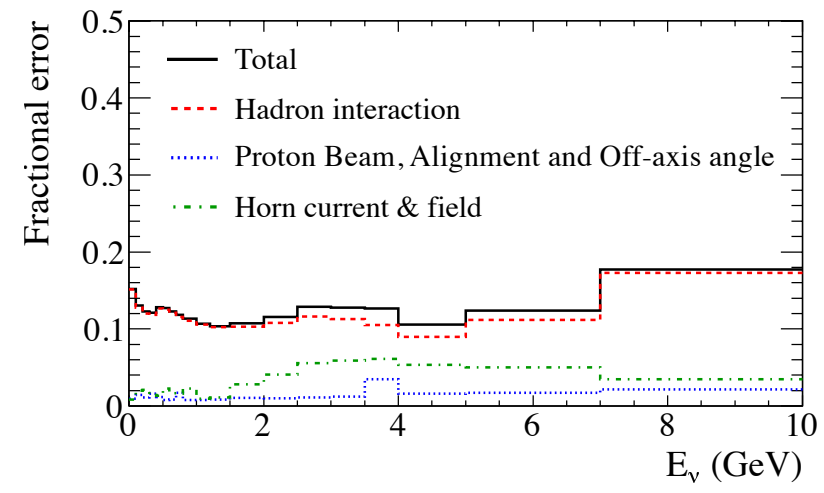

FIG. 5. Fractional flux error at module 3, including all sources of uncertainty.

\section{B. Neutrino-nucleus interaction simulation}

Neutrino-nucleus (Fe) interactions in the INGRID module are simulated by a neutrino event generator, which is a composite of different neutrino interaction models. NEUT (ver.5.1.4.2) [18] is used as the primary event generator in this analysis. GENIE (ver.2.8.0) [19], a different neutrino interaction simulation package, is also used for comparison. This section first describes the various interaction models simulated in the NEUT event generator, then explains the systematic uncertainties associated with each model. Details of the event generators used in T2K can be found in Ref. [20].

\section{The NEUT neutrino event generator}

Given a neutrino energy and a detector geometry, NEUT determines the interaction mode of an event and calculates the kinematics of the final state particles. It also simulates FSI for hadrons as they traverse the target nucleus. The following interaction modes are provided for both charged-current (CC) and neutral-current (NC) interactions by NEUT:

- Quasi-elastic scattering (CCQE or NCQE)

- Resonant pion production ( $\mathrm{CC} 1 \pi$ or $\mathrm{NC} 1 \pi)$

- Deep inelastic scattering (CCDIS or NCDIS)

- Coherent pion production

Here $N$ and $N^{\prime}$ denote nucleons, $l$ is the lepton, and $A$ is the target nucleus.

\section{Neutrino interaction model uncertainties}

Table II summarizes the parameters used for modeling neutrino interactions in NEUT. The systematic parameters listed in the table were evaluated in the previous analyses of neutrino oscillation from $\mathrm{T} 2 \mathrm{~K}$ [21, 22] and fall into the following four categories:
$M_{A}^{Q E}, M_{A}^{R E S}$, and the nuclear model:

These parameters are used for modeling CCQE and $\mathrm{CC} 1 \pi$ interactions. A $20 \%$ error is assigned to the axial vector masses, which comes from a comparison of external measurements of these parameters. The uncertainty on the Fermi momentum and binding energy are estimated from electron scattering data [23]. The uncertainty in the nuclear model is evaluated by exchanging the RFG nuclear model with the spectral function model described in Ref. [24].

\section{$\pi$-less $\Delta$ decay and $\mathrm{W}$ shape:}

In the resonant pion production process, baryon resonances, mainly $\Delta \mathrm{s}$, can interact with other nucleons in the target nucleus, and disappear without pion emission. The $\pi$-less $\Delta$ decay parameter is introduced to take into account the uncertainties in this process. The $\mathrm{W}$ shape parameter is introduced to modify the shape of the momentum distribution of pions from NC single pion production interactions so that it matches MinibooNE data [25].

\section{Normalization parameters:}

Normalization parameters are used to change the overall normalization of the cross section. The normalizations for $\mathrm{CCQE}$ and $\mathrm{CC} 1 \pi$ are defined separately for different energy regions. The uncertainties on the normalizations are mostly determined from the MiniBooNE data. The CC other shape parameter is introduced as an energy dependent uncertainty on CC DIS and CC resonant interactions, where the resonance decays into a nucleon and photon, kaon or eta. According to the MINOS measurement [1], the relative uncertainty on the CC inclusive cross section on iron, which is dominated by CCDIS, is approximately $10 \%$ at $4 \mathrm{GeV}$. Using this as a reference point, the error on the CCDIS and CC resonant cross section is scaled using the following formula:

$$
\frac{\delta \sigma_{C \text { Cother }}}{\sigma_{C \text { Cother }}}=\frac{0.4 \mathrm{GeV}}{E_{\nu}(\mathrm{GeV})}
$$

Although the error goes to infinity as $E_{\nu}$ approaches $0 \mathrm{GeV}$, this error does not have a significant contribution to the total cross section error for the lower neutrino energy region because the interactions have a threshold energy of approximately $0.6 \mathrm{GeV}$ and a small cross section in the $1 \mathrm{GeV}$ energy region. Finally, the $1 \pi E_{\nu}$ shape parameter is a weighting factor as a function of neutrino energy. This is introduced to cover the discrepancy between the MiniBooNE measurement of the $\mathrm{CC} 1 \pi$ cross section versus $E_{\nu}$ and the NEUT prediction using the best fit parameters obtained from the fit to the MinibooNE data [26]. This discrepancy is as large as $50 \%$ at $600 \mathrm{MeV}$, so an error of $50 \%$ is assigned to this weighting factor. In the nominal 
TABLE II. Neutrino interaction systematic parameters, nominal values, uncertainties $(1 \sigma)$, and interaction types $(\mathrm{CC}$, NC or $\mathrm{CC}+\mathrm{NC})$. The first, second, and third groups represent the model parameters, the ad hoc parameters, and the pion FSI parameters respectively. A 1 or 0 in the nominal value column means that the effect of the systematic parameter is implemented or not implemented by default [21, 22].

\begin{tabular}{|c|c|c|c|}
\hline Parameter & Nominal value & Uncertainty $(1 \sigma)$ & Interaction type \\
\hline$M_{A}^{Q E}$ & $1.21 \mathrm{GeV}$ & $0.20 \mathrm{GeV}$ & $\mathrm{CC}$ \\
\hline$M_{A}^{R E S}$ & $1.21 \mathrm{GeV}$ & $0.20 \mathrm{GeV}$ & $\mathrm{CC}+\mathrm{NC}$ \\
\hline Fermi momentum (Fe) & $250 \mathrm{MeV} / \mathrm{c}$ & $30 \mathrm{MeV} / \mathrm{c}$ & $\mathrm{CC}$ \\
\hline Binding energy $(\mathrm{Fe})$ & $33 \mathrm{MeV}$ & $9 \mathrm{MeV} / \mathrm{c}$ & $\mathrm{CC}$ \\
\hline Spectral function & 0 (off) & 1 (on) & $\mathrm{CC}$ \\
\hline$\pi$-less $\Delta$ decay & 0.2 & 0.2 & $\mathrm{CC}+\mathrm{NC}$ \\
\hline W shape & $87.7 \mathrm{MeV}$ & $45.3 \mathrm{MeV}$ & $\mathrm{CC}+\mathrm{NC}$ \\
\hline CCQE normalization $\left(E_{\nu} \leq 1.5 \mathrm{GeV}\right)$ & 1 & 0.11 & $\mathrm{CC}$ \\
\hline CCQE normalization $\left(1.5 \leq E_{\nu} \leq 3.5 \mathrm{GeV}\right)$ & 1 & 0.30 & $\mathrm{CC}$ \\
\hline CCQE normalization $\left(E_{\nu} \geq 3.5 \mathrm{GeV}\right)$ & 1 & 0.30 & $\mathrm{CC}$ \\
\hline CC1 $\pi$ normalization $\left(E_{\nu} \leq 2.5 \mathrm{GeV}\right)$ & 1 & 0.21 & $\mathrm{CC}$ \\
\hline CC1 $\pi$ normalization $\left(E_{\nu} \geq 2.5 \mathrm{GeV}\right)$ & 1 & 0.21 & $\mathrm{CC}$ \\
\hline CC coherent normalization & 1 & 1.0 & $\mathrm{CC}$ \\
\hline CC other shape & 0 & 0.1 at $E_{\nu}=4.0 \mathrm{GeV}$ & $\mathrm{CC}$ \\
\hline NC $1 \pi^{0}$ normalization & 1 & 0.31 & $\mathrm{NC}$ \\
\hline NC coherent pi normalization & 1 & 0.30 & $\mathrm{NC}$ \\
\hline NC $1 \pi^{ \pm}$normalization & 1 & 0.30 & $\mathrm{NC}$ \\
\hline NC other normalization & 1 & 0.30 & $\mathrm{NC}$ \\
\hline $1 \pi E_{\nu}$ shape & 0 (off) & 0.50 & $\mathrm{CC}+\mathrm{NC}$ \\
\hline Pion absorption & 1 & 0.5 & $\mathrm{CC}+\mathrm{NC}$ \\
\hline Pion charge exchange $\left(P_{\pi}<500 \mathrm{MeV} / \mathrm{c}\right)$ & 1 & 0.5 & $\mathrm{CC}+\mathrm{NC}$ \\
\hline Pion charge exchange $\left(P_{\pi}>400 \mathrm{MeV} / \mathrm{c}\right)$ & 1 & 0.3 & $\mathrm{CC}+\mathrm{NC}$ \\
\hline Pion QE scattering $\left(P_{\pi}<500 \mathrm{MeV} / \mathrm{c}\right)$ & 1 & 0.5 & $\mathrm{CC}+\mathrm{NC}$ \\
\hline Pion QE scattering $\left(P_{\pi}>400 \mathrm{MeV} / \mathrm{c}\right)$ & 1 & 0.3 & $\mathrm{CC}+\mathrm{NC}$ \\
\hline Pion inelastic scattering & 1 & 0.5 & $\mathrm{CC}+\mathrm{NC}$ \\
\hline
\end{tabular}

NEUT, this weighting is not applied ("off" as in Table II).

\section{Pion FSI:}

There are three pion FSI processes of interest in the T2K energy range: absorption, charge exchange and QE scattering. In addition to these interactions, the particle production process, defined as "inelastic scattering", was considered, since it is the dominant process at higher pion energies. Uncertainties on the FSI parameters are estimated using external data sets [27].

Propagation of these uncertainties is described in Sec. VB.

\section{Detector simulation}

The particle type and kinematic information provided by NEUT is passed to the detector simulation built within a GEANT4 framework [28]. All the detector components are modeled in the simulation. The energy deposited by each particle in the scintillator planes is converted into a number of photo-electrons, taking into account the non-linear response of the scintillator, light collection efficiency and attenuation of the WLS fiber, and the non-linearity of the MPPC response. The non-linear response of the ADCs on the front-end electronics is also modeled based on the results of charge injection tests.

Particles generated in the wall upstream of the INGRID detectors are also propagated into the detector simulation, and are treated as a background (BG) source.

The hadronic interaction of particles in the detector is simulated by GEANT4 using the FTFP_BERT physics list. In this physics list, the GEANT4 Bertini cascade model is used to simulate nuclear reactions by hadrons with kinetic energies below $5.5 \mathrm{GeV}$. For particles with kinetic energies above $5 \mathrm{GeV}$, the list uses the Fritiof model $[29,30]$.

\section{ANALYSIS METHOD}

\section{A. Overview}

First, neutrino interactions are selected in each INGRID module. The different neutrino energy spectra sampled by the different modules provide a way of extracting the energy dependent cross section. The selected events are further categorized according to the topology of their final-state muon in order to improve the sensitivity of the samples to the energy of the incoming neutrino. A PDF is then constructed relating the different INGRID modules and event topologies to the neutrino energy. Finally, to extract the $\mathrm{CC}$ inclusive cross section, a $\chi^{2}$ fit 
is performed between the selected events and the PDFs.

\section{B. Neutrino event selection}

A detailed description of the event selection for neutrino interactions at INGRID can be found in Refs. [2, 31]. A brief explanation of each step of the selection is given here. A typical selected muon-neutrino interaction candidate is shown in Fig. 6.
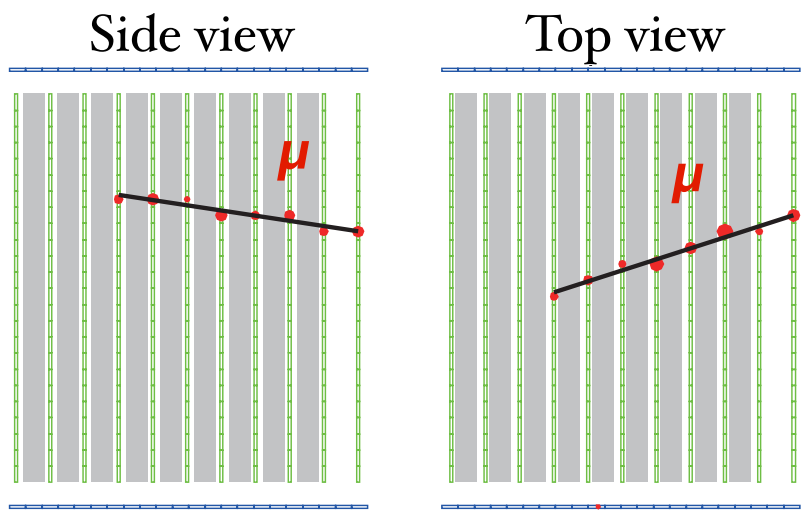

FIG. 6. Event display of a muon-neutrino event candidate. Circles and solid lines represent the hits at the scintillator planes and reconstructed tracks, respectively. The neutrino beam enters from the left side.

\section{Pre-selection:}

The integrated charge and timing of hits in each channel are recorded with a 2.5 photo-electron threshold. If there are more than 3 hit channels within a 100 nsec time window, these hits are combined to form a hit cluster. The scintillator planes are then searched to find those with at least one hit in both their X and Y oriented layers. Such a plane is called an "active plane", and events with 2 or more active planes are selected.

\section{2D track reconstruction:}

A "cellular automaton" tracking algorithm [32] is applied to hits in the $\mathrm{X}$ and $\mathrm{Y}$ planes to obtain tracks in the $\mathrm{XZ}$ view and $\mathrm{YZ}$ view respectively.

\section{3D track matching:}

The difference in the most upstream layer hit between any two tracks in the $\mathrm{XZ}$ view and $\mathrm{YZ}$ view is used to determine if they originate from the same vertex. If this difference is greater than 2 planes then the tracks are not matched.

\section{Vertexing:}

The vertex is defined as the most upstream hit of the track. If there are two or more 3D tracks, a check is performed to see if they originate from the same vertex or not.

\section{Timing cut:}

The T2K neutrino beam has eight bunches in each beam spill, and each bunch has a width of 58 nsec. The selected events are required to lie within $100 \mathrm{nsec}$ of the expected time of each bunch.

\section{Veto cut:}

The reconstructed track is extrapolated to upstream positions in the side veto planes and upstream veto planes, and the event is cut if any hits are found near the extrapolated entry point.

\section{Fiducial volume cut:}

The fiducial volume $(\mathrm{FV})$ is defined as a cube with a $( \pm 50) \times( \pm 50) \mathrm{cm}^{2}$ transverse area, corresponding to the scintillator bars from the 3rd to 22nd channel in the $\mathrm{X}$ and $\mathrm{Y}$ direction, and from tracking plane\#1 to 8 in the $\mathrm{Z}$ direction (see Fig. 7). Events with a vertex inside the FV are selected.
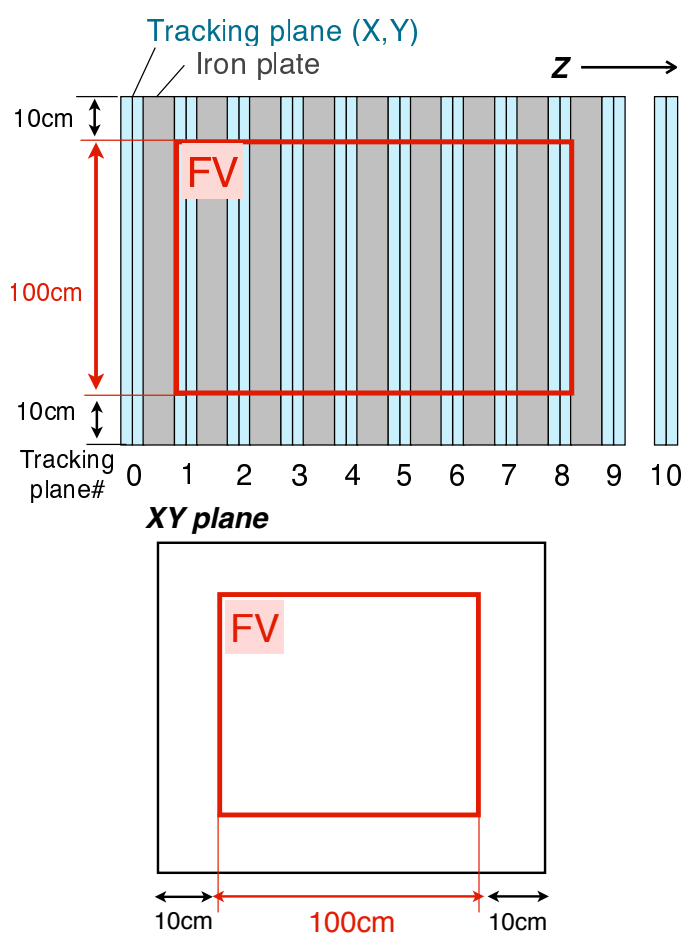

FIG. 7. Fiducial volume of the INGRID module viewed from of the side (top) and front (bottom).

After the event selection above, corrections are applied to the $\mathrm{MC}$ to account for differences in the individual iron target masses, the observed background rate, and the number of dead channels. We also apply a correction for event pileup, which depends on the beam intensity and results in a loss of efficiency [31]. Table III summarizes the number of events predicted by the MC simulation and observed in the data.

The selected events contain the muon-neutrino signal as well as background events, such as $\bar{\nu}_{\mu}$ and $\nu_{e}$ inter- 
TABLE III. Summary of the neutrino event selection. The p.o.t. used in the MC simulation is normalized to correspond to the data set used in this analysis, i.e. $6.27 \times 10^{20}$.

\begin{tabular}{l|c|cccc}
\hline \hline & Data & \multicolumn{4}{|c}{ MC } \\
& & $\nu_{\mu}$ & $\bar{\nu}_{\mu}+\nu_{e}$ & beam-related BG & Total \\
\hline Vertexing & $3.993 \times 10^{7}$ & $1.655 \times 10^{7}$ & $0.039 \times 10^{7}$ & $2.294 \times 10^{7}$ & $3.987 \times 10^{7}$ \\
Timing cut & $3.992 \times 10^{7}$ & $1.655 \times 10^{7}$ & $0.039 \times 10^{7}$ & $2.294 \times 10^{7}$ & $3.987 \times 10^{7}$ \\
Veto cut & $1.725 \times 10^{7}$ & $1.458 \times 10^{7}$ & $0.036 \times 10^{7}$ & $0.239 \times 10^{7}$ & $1.733 \times 10^{7}$ \\
FV cut & $1.103 \times 10^{7}$ & $1.098 \times 10^{7}$ & $0.027 \times 10^{7}$ & $0.006 \times 10^{7}$ & $1.131 \times 10^{7}$ \\
\hline \hline
\end{tabular}

actions. The other backgrounds come from muons, neutrons and photons generated by neutrino interactions out of the FV or in the pit wall upstream of INGRID (hereafter called beam-related BG). Since the contamination of $\bar{\nu}_{e}$ is negligible, it is not counted in the MC. The angular distribution of the lepton track for the selected events is shown in Fig. 8. Since the vertex is defined as the most upstream hit of the track, the angular acceptance is limited to between $0^{\circ}$ and $90^{\circ}$.

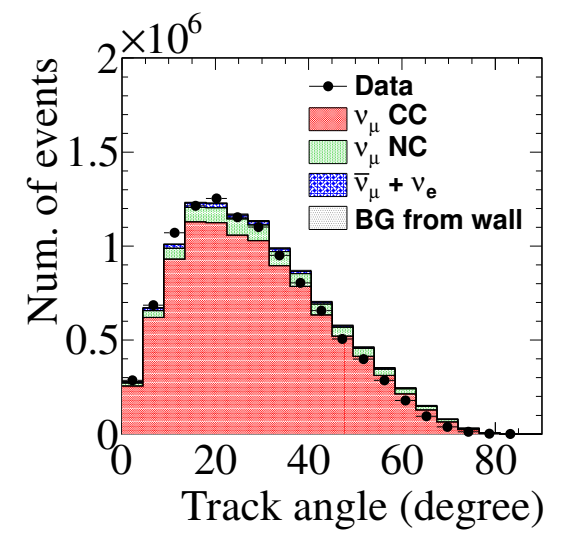

FIG. 8. Distribution of the reconstructed track angle with respect to the beam direction after the event selection. The number of events shown in the figure is the total integrated over all modules.

The final selected event sample has $>70 \%$ efficiency for $\mathrm{CC}$ interactions from neutrinos with energies $>1 \mathrm{GeV}$, as shown in Fig. 9. Around 5\% of the selection inefficiency in the higher energy region is due to events where the muon is produced at a large angle to the $\mathrm{Z}$ axis of the detector. This results in it escaping the module before passing through two active planes. The predicted energy spectrum of the reconstructed $\nu_{\mu}$ events at the INGRID modules is shown in Fig. 10.

\section{Event topology}

To improve the sensitivity of this analysis to the energy of the neutrino, the selected events are categorized into the following two topologies:

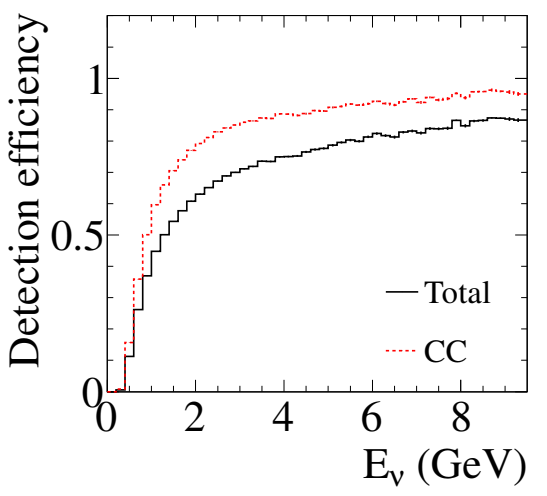

FIG. 9. Detection efficiency for CC+NC (solid line) and CC (dashed line) events. These efficiency curves are estimated from the number of events integrated over all modules.

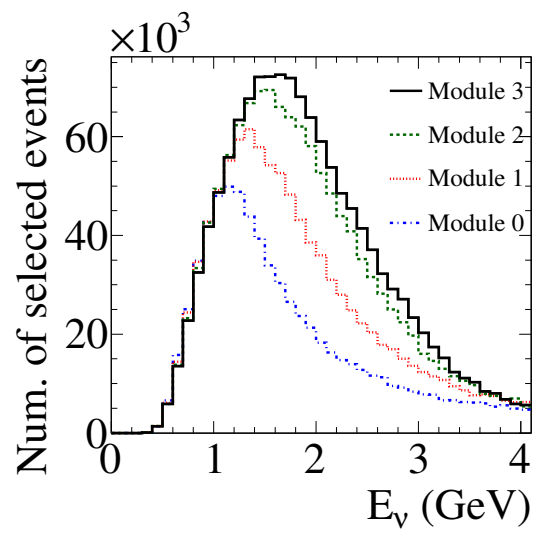

FIG. 10. Predicted energy spectrum of the reconstructed events at the different INGRID modules.

\section{Downstream (DS-) escaping}

\section{Non-downstream (NonDS-) escaping}

If one of the tracks from the neutrino interaction penetrates the most downstream plane, as shown on the left in Fig. 11, that event is categorized as DS-escaping. All other events, i.e. both side escaping and fully contained 
events (see the right plot in Fig. 11) are categorized as NonDS-escaping.
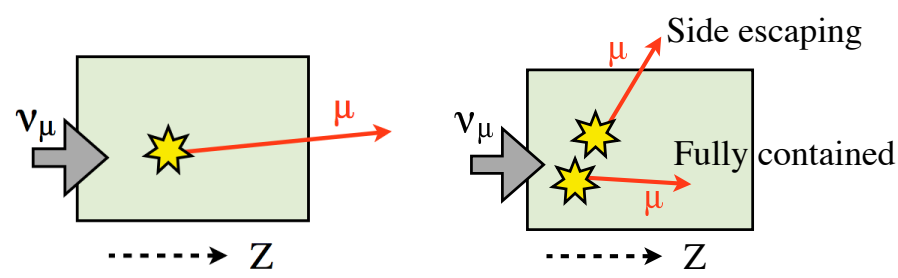

FIG. 11. Different event topologies. If a track penetrates the most downstream plane, the event is categorized as DSescaping (left). The other events are categorized as NonDSescaping (right).

Events are then further categorized according to the reconstructed vertex-Z position. Vertex-Z is defined as the most upstream active plane number and ranges from 1 (most upstream) to 8 (most downstream). Events whose vertex- $Z$ is in the most downstream plane are greatly affected by uncertainties in the GEANT4 hadron production model, so only events with vertex- $\mathrm{Z}$ in the range 1-7 are used in this analysis.

In total, there are 14 event topologies:

- DS-escaping: vertex-Z=1-7

- NonDS-escaping: vertex-Z=1-7

Figure 12 shows the energy spectra of "DS-escaping \& vertex-Z $=1$ " events and "NonDS-escaping \& vertex$\mathrm{Z}=7$ " events for module 0 . The former has a more energetic $\mu$ track and are generally produced by higher energy neutrinos. The latter, on the other hand, tend to have muons produced at a larger angle to the neutrino beam or with a lower energy, and so the majority come from lower energy neutrinos.

\section{Module grouping}

A shift of the neutrino beam direction changes the peak of the neutrino energy spectra at the INGRID modules. In order to reduce this effect, for the horizontal and vertical directions separately, the two modules at beam-axis symmetric positions are grouped together. This results in 7 module groups in total, defined in Table IV.

The number of selected events for each module group and each topology is then defined as:

$$
N_{j g}=\frac{N_{j m}+N_{j m^{\prime}}}{2}
$$

where the indices $j$ and $g$ denote the $j^{\text {th }}$ topology and the $g^{t h}$ module group $(g=1,2, . ., 7)$, respectively. The $m$ and $m^{\prime}$ indices stand for the module numbers corresponding to each module group.

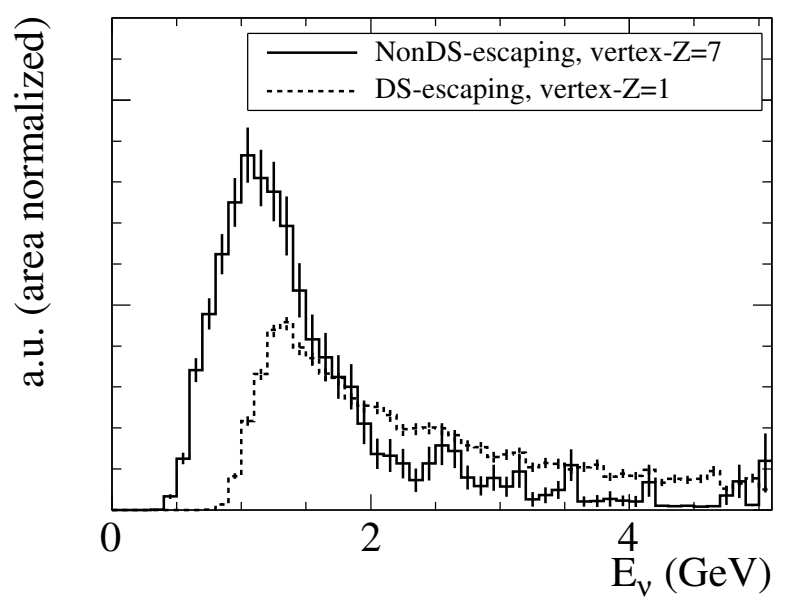

FIG. 12. Neutrino energy spectra for "NonDS-escaping \& vertex-Z=7" (solid line) events and "DS-escaping \& vertex$\mathrm{Z}=1$ " (dashed line) events. The energy spectra for module 0 are shown, normalized by area.

TABLE IV. Definition of the module groups

\begin{tabular}{|c|c|c|c|c|}
\hline $\begin{array}{l}\text { Module } \\
\text { group }\end{array}$ & $\begin{array}{c}\text { Module } \\
\text { ID }\end{array}$ & $\begin{array}{c}\text { Distance from } \\
\text { the beam axis }(\mathrm{cm})\end{array}$ & $\begin{array}{c}\text { Off-axis } \\
\text { angle }\end{array}$ & $\begin{array}{c}\text { Horizontal or } \\
\text { Vertical }\end{array}$ \\
\hline 1 & 0,6 & 450 & $1.1^{\circ}$ & Horizontal \\
\hline 2 & 7,13 & 450 & $1.1^{\circ}$ & Vertical \\
\hline 3 & 1,5 & 300 & $0.7^{\circ}$ & Horizontal \\
\hline 4 & 8,12 & 300 & $0.7^{\circ}$ & Vertical \\
\hline 5 & 2,4 & 150 & $0.4^{\circ}$ & Horizontal \\
\hline 6 & 9,11 & 150 & $0.4^{\circ}$ & Vertical \\
\hline 7 & 3,10 & 0 & $0^{\circ}$ & (Center) \\
\hline
\end{tabular}

\section{E. Detector response uncertainties}

This section introduces two different kind of detector response uncertainty: those producing correlation among the event topologies and those that do not. They are summarized as follow:

- Uncorrelated error sources

- Mass of iron plate

- Pileup correction

- Correlated error sources

- Event selection and reconstruction

- MPPC noise rate

The treatment of these systematic uncertainties in this analysis is described in Sec. V 


\section{Uncorrelated errors}

\section{Iron mass:}

The error on the measurement of the mass of each iron plate and the machining tolerance for the plate area are taken into account in the systematic error on the iron mass. Since these errors are independent for each iron plate, an uncorrelated error of $0.09 \%$ assigned to the number of selected events.

\section{Pileup correction:}

The number of selected events is corrected to account for event pileup. The correction factor is estimated using data sets at different beam intensities. The uncertainty on the correction factor comes from the statistical error on the number of events, so the uncertainty is uncorrelated between the event topologies. An error of 0.5-2.0\%, depending on the event topology, is assigned in this analysis.

\section{Correlated errors}

\section{MPPC noise:}

MPPC noise hits sometimes result in misreconstruction of the event vertex or a miscounting of the number of active planes, which produces a variation in the neutrino event selection efficiency. The systematic error caused by the variation in the measured noise rate over time is evaluated by altering the noise rate in the MC. As a result, a $0.1-1 \%$ error is assigned to the number of selected event in each topology.

\section{Event selection:}

In this analysis, uncertainties in the following event selection steps are taken into account:

- 3D track matching

- Vertexing

- Veto cut

- FV cut

The systematic error on the number of selected events is evaluated by varying the selection threshold for each step, picking the loosest or tightest threshold. The change from the nominal threshold to the loosest (tightest) is defined as the $+1 \sigma(-1 \sigma)$ change. The resultant fractional variation in the number of selected events $(\equiv \Delta N / N)$ due to the $\pm 1 \sigma$ change is computed for both the data and MC. Any difference in $\Delta N / N$ between the data and $\mathrm{MC}$ is then taken as a systematic error.

Uncertainties on the hit efficiency of the tracking planes, the contamination due to beam-related BGs, the hit inefficiency of the upstream veto plane, and the tracking efficiency were found to be negligible for this analysis and are not included in the final result.

\section{F. Cross-section extraction}

This analysis uses the least $\chi^{2}$ method to fit to the observed number of events at each module group $\left(g^{\text {th }}\right.$ bin: 1-7) and for each event topology ( $j^{\text {th }}$ bin: 1-14):

$$
\begin{aligned}
\chi^{2}= & \sum_{j} \sum_{g} \frac{\left\{N_{j g}^{o b s}-\left(N_{j g}^{c c}+N_{j g}^{n c}+N_{j g}^{b g}\right)\right\}^{2}}{\left(\sigma_{N_{j g}}\right)^{2}} \\
& +\sum_{k} \Delta\left(\vec{f}^{k}\right)^{t}\left(V_{k}\right)^{-1} \Delta \vec{f}^{k}
\end{aligned}
$$

where $N^{o b s}$ is the observed number of events, $N^{c c}, N^{n c}$, and $N^{b g}$ are the expected numbers of CC events, NC, and BG events, and $\Delta \vec{f}_{k}$ and $V_{k}$ are the systematic parameter and the covariance for the $k^{t h}$ error source, respectively. For the covariance term uncertainties in the neutrino flux, neutrino interaction model and the detector response are taken into account and are described in Sec. V. The denominator in the $\chi^{2}$ statistical term is composed of the statistical error on the observed number of events $\left(N_{j g}^{o b s}\right)$, the MC statistical error $\left(\sigma_{N_{j g}^{m c}}\right)$, and the error on the detector response, which is uncorrelated among event topologies: $\left(\sigma_{N_{j g}^{\text {det }}}\right)$ :

$$
\sigma_{N_{j g}}=\sqrt{N_{j g}^{o b s}+\left(\sigma_{N_{j g}^{m c}}\right)^{2}+\left(\sigma_{N_{j g}^{d e t}}\right)^{2}} .
$$

The expected number of CC events in the $g^{\text {th }}$ module group and for the $j^{\text {th }}$ event topology is expressed as:

$$
\begin{aligned}
N_{j g}^{c c} \simeq \sum_{i}[ & \left(1+\Delta f_{j}^{d}+\Delta f_{j}^{c c}+\Delta f_{i g}^{b}+\Delta f_{i}\right) \\
& \left.\times \phi_{i g} \cdot \sigma_{i}^{c c} \cdot \epsilon_{i j}^{c c} \cdot T\right]
\end{aligned}
$$

- $\Delta f^{d}$ : systematic parameter for the detector response,

- $\Delta f^{c c}$ : systematic parameter for the CC interaction model,

- $\Delta f^{b}$ : systematic parameter for the $\nu_{\mu}$ flux,

- $\phi \quad: \nu_{\mu}$ flux,

- $\sigma^{c c}: \nu_{\mu}$ CC cross section,

- $\epsilon^{c c}$ : detection efficiency for the CC interaction,

- $T$ : the number of nucleons

in the fiducial volume of the INGRID module.

Here $i$ goes over the neutrino energy bins, described in Sec. IV G, and $\Delta f_{i}$ is the parameter being fit, which is used to represent fractional deviations of the CC inclusive cross section. The systematic parameters, $\Delta f_{j}^{d}, \Delta f_{j}^{c c}$ and $\Delta f_{i g}^{b}$, are also fit to include the effect of these systematics into the cross-section result. The INGRID modules are formed from both iron and plastic scintillator $(\mathrm{CH})$. The effect on this result coming from the different target nucleus for $\mathrm{CH}$ interactions is found to be small, so the event rate per unit weight on $\mathrm{CH}$ is assumed to be equal 
to that on iron. $\Delta f_{j}^{d}$ and $\Delta f_{j}^{c c}$ are systematic parameters representing the uncertainty on the detector response and the CC cross-section model respectively for the $j^{\text {th }}$ topology bin. These uncertainties change the detection efficiency as a function of neutrino energy, resulting in a variation in the number of selected events. The difference in these uncertainties between the module groups is very small, therefore the same parameters are applied to all module groups. $\Delta f_{i g}^{b}$ parameterizes the flux uncertainty, changing the normalization of the neutrino flux, in the $i^{t h}$ energy bin of the $g^{t h}$ module. The $\Delta f_{j}^{d}, \Delta f_{j}^{c c}$ and $\Delta f_{i g}^{b}$ parameters describe fractional deviations from the nominal $\mathrm{MC}$ and change the number of events in each event topology, module group, and energy bin.

Since the fraction of NC events in the selected sample is very small, the NC events are summed over the entire energy region and an averaged flux systematic parameter is applied to them:

$$
\Delta \bar{f}_{g}^{b}=\sum_{i} \Delta f_{i g}^{b} \cdot \frac{\phi_{i g}}{\sum_{i^{\prime}} \phi_{i^{\prime} g}} .
$$

We express the number of the NC events as follows:

$$
N_{j g}^{n c} \simeq \sum_{i}\left(1+\Delta f_{j}^{d}+\Delta \bar{f}_{g}^{b}+\Delta f_{j}^{n c}\right) \cdot \phi_{i g} \cdot \sigma_{i}^{n c} \cdot \epsilon_{i j}^{n c} \cdot T .
$$

The other BG events (the beam related BG and $\bar{\nu}_{\mu}$ and $\nu_{e}$ beam flux components) are summed for each module and for each topology $\left(N_{j g}^{b g}\right)$. The number of BG events in the sample, and their associated errors, are both small, so the errors on these BGs are neglected in this analysis.

\section{G. Energy binning}

The cross section is required to be continuous at the bin boundaries and is linearly interpolated between boundaries, so Eq. (6) is modified as follows:

$$
\begin{aligned}
N_{j g}^{c c}=\left(1+\Delta f_{j}^{d}\right) \cdot \sum_{i}\left(1+\Delta f_{i g}^{b}\right) \\
\times \sum_{l=0}^{L_{i}}\left[\left(1+\Delta f_{i}+\frac{\Delta f_{i+1}-\Delta f_{i}}{L_{i}} \cdot l\right)\right. \\
\left.\times \phi_{i l g} \cdot \sigma_{i l}^{c c} \cdot \epsilon_{i l j}^{c c} \cdot T\right] .
\end{aligned}
$$

To interpolate, each $i^{\text {th }}$ energy bin is divided into fine bins $\left(l=0,1, . ., L_{i}\right)$. The $i^{t h}$ energy bin is defined as the "global bin" and the $l^{\text {th }}$ energy bin as the "local bin", respectively. The energy range of the global bins and the binning for the local bins are summarized in Table V. The $\Delta f_{i}$ s are set at $0.5,0.8,1.4,2.6$, and $4.0 \mathrm{GeV}$.

Finally, the energy dependent CC inclusive cross sections are extracted as follows. After deriving the fit parameters using the least $\chi^{2}$ method, the cross sections are obtained by multiplying those in the original model by
TABLE V. Summary of the energy range of each global bin, the size of each local bin, and the number of the local bins per global bin.

\begin{tabular}{ccc}
\hline \hline $\begin{array}{c}\text { Energy range of } \\
\text { global bin }(\mathrm{GeV})\end{array}$ & $\begin{array}{c}\text { Size of each } \\
\text { local bin }(\mathrm{MeV})\end{array}$ & $\begin{array}{c}\text { Number of } \\
\text { local bins }\left(L_{i}\right)\end{array}$ \\
\hline $0-0.5$ & 500 & 1 \\
$0.5-0.8$ & 100 & 3 \\
$0.8-1.4$ & 100 & 6 \\
$1.4-2.6$ & 100 & 12 \\
$2.6-4.0$ & 100 & 14 \\
$4.0-30.0$ & 26000 & 1 \\
\hline \hline
\end{tabular}

$1+\frac{\Delta f_{1}+\Delta f_{2}}{2}, 1+\frac{\Delta f_{2}+\Delta f_{3}}{2}$, and $1+\frac{\Delta f_{3}+\Delta f_{4}}{2}$. Taking the average of neighboring parameters produces a measurement at the central energy between the bin boundaries, since, as in Eq. (9), a linear interpolation is applied between the neighboring $\Delta f_{i}$ parameters. As a result of this averaging, the cross section is measured at 1.1, 2.0, and $3.3 \mathrm{GeV}$. The final error on the cross section is smaller than those on the $\Delta f_{i}$ parameters due to the negative correlations between the $\Delta f_{i}$ 's. This feature is a result of the cross section continuity requirement.

As seen in Fig. 9, the INGRID detection efficiency for $\mathrm{CC}$ interactions falls rapidly for neutrinos with energies less than $0.5 \mathrm{GeV}$. Since the event samples are not sensitive to the cross section in this region $\Delta f_{0}$ is not used in the final result. For $E_{\nu}>4.0 \mathrm{GeV}$ there is only a small difference in the neutrino energy spectra between the INGRID modules. Therefore, sensitivity to the cross section for $E_{\nu}>4.0 \mathrm{GeV}$ is expected to be worse compared to the lower energy regions. For these reasons it was decided, before fitting the data, to use $\Delta f_{1}-\Delta f_{4}$ to measure the cross section at $1.1,2.0$, and $3.3 \mathrm{GeV}$.

The PDF of CC events in the global energy binning is shown in Fig. 13. Here, the "Fraction" described by the $\mathrm{Z}$-axis of the figure is obtained for each energy region by dividing the number of $\mathrm{CC}$ events in each bin by the total number of $\mathrm{CC}$ events in that energy regions. At lower neutrino energies most of the $\mathrm{CC}$ events are selected in the downstream vertex-Z bin for the DS-escaping topology whereas at higher energies the DS-escaping events are distributed uniformly in vertex-Z. NonDS-escaping $\mathrm{CC}$ events are selected in all vertex-Z bins for low energy neutrinos but higher energy neutrinos tend to be located in upstream vertex-Z bins. In addition, more highenergy neutrino events are selected in modules closer to the beam-axis.

\section{PROPAGATION OF SYSTEMATIC UNCERTAINTIES}

As described in Sec. IV F, the $\chi^{2}$ has terms with covariance matrices for systematic parameters. In this section, 
NonDS-escaping, 0.5-0.8 GeV

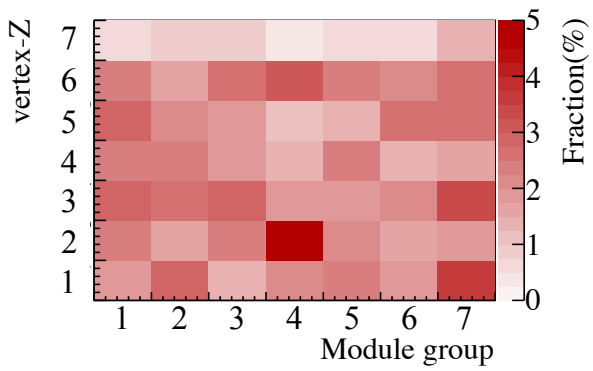

NonDS-escaping, $0.8-1.4 \mathrm{GeV}$

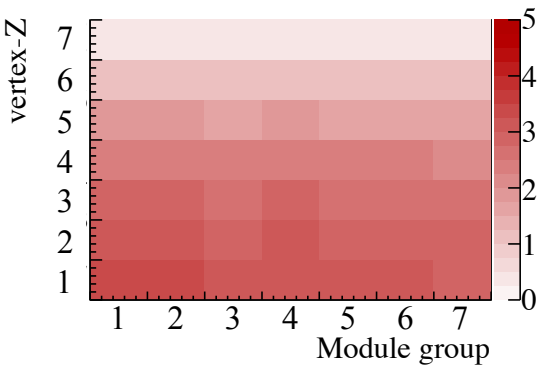

NonDS-escaping, 1.4-2.6 GeV

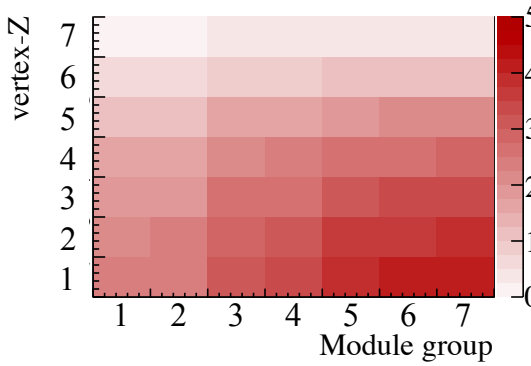

NonDS-escaping, 2.6-4.0 GeV

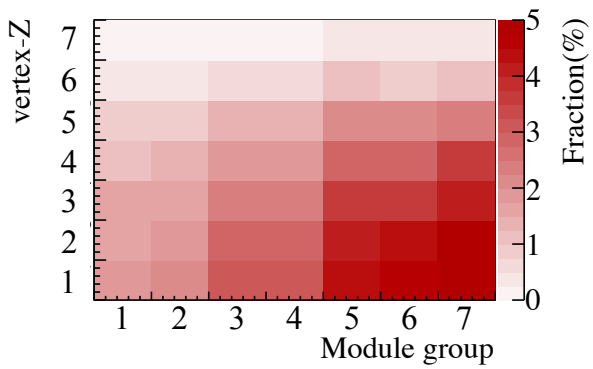

NonDS-escaping, $\geq 4 \mathrm{GeV}$

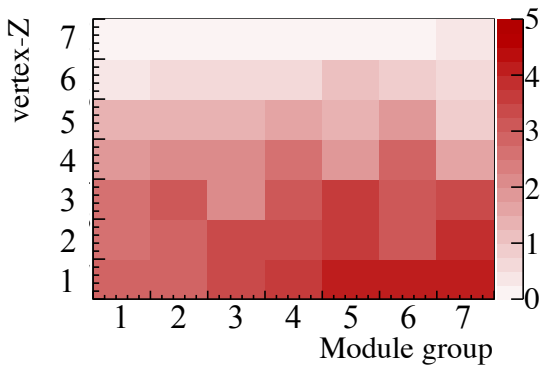

DS-escaping, 0.5-0.8 GeV

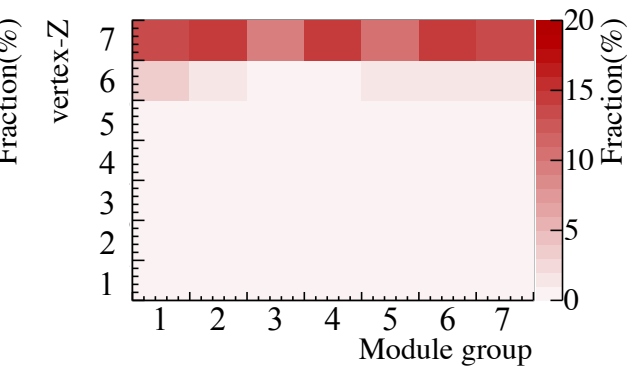

DS-escaping, 0.8-1.4 GeV

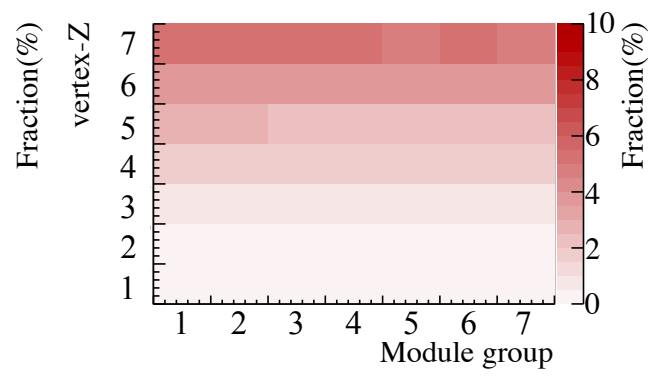

DS-escaping, 1.4-2.6 GeV

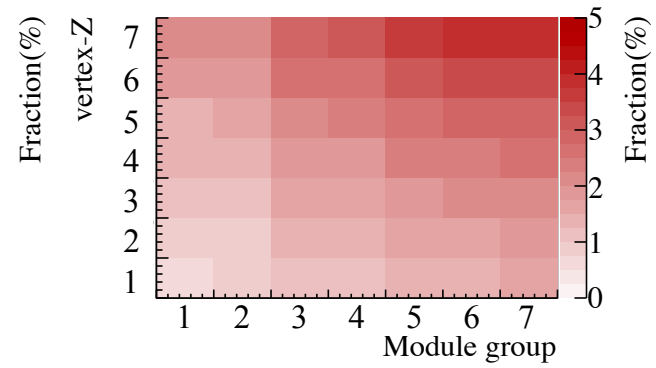

DS-escaping, 2.6-4.0 GeV

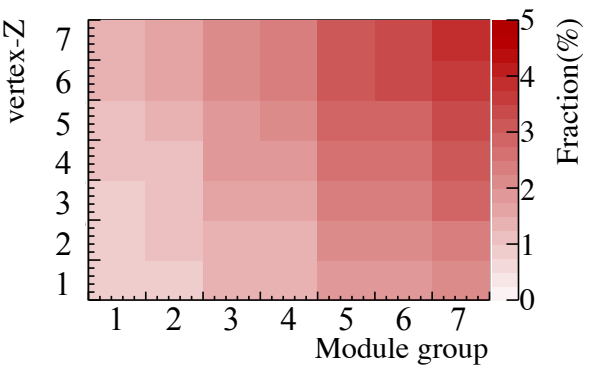

DS-escaping, $\geq 4 \mathrm{GeV}$

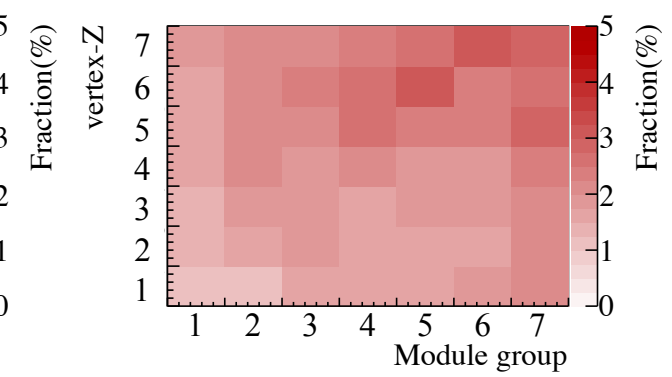

FIG. 13. Probability density function (PDF) for each energy region defined in Sec. IV G: $E_{\nu}=0.5-0.8 \mathrm{GeV}, 0.8-1.4 \mathrm{GeV}$, 1.4-2.6 GeV, 2.6-4.0 GeV, >4.0 GeV. 
we describe how the covariance matrices for the neutrino flux, the neutrino interaction model and the detector response, which were introduced in Secs. III A, III B and IV E, respectively, are constructed.

\section{A. Neutrino flux uncertainties}

The covariance matrix for each source of error on the neutrino flux perdiction, such as the horn current uncertainty, is calculated by taking the variation of the flux due to that error. The total covariance matrix is obtained by summing all these matrices and Figure 14 shows the correlation matrix derived from it. The energy binning in the covariance matrix is the same as that used to define the "global bin" in Table V. One can see that it is largely positively correlated. This correlation comes mainly from the uncertainty associated with hadron production at the target, which varies the neutrino flux in the same way at all INGRID modules.

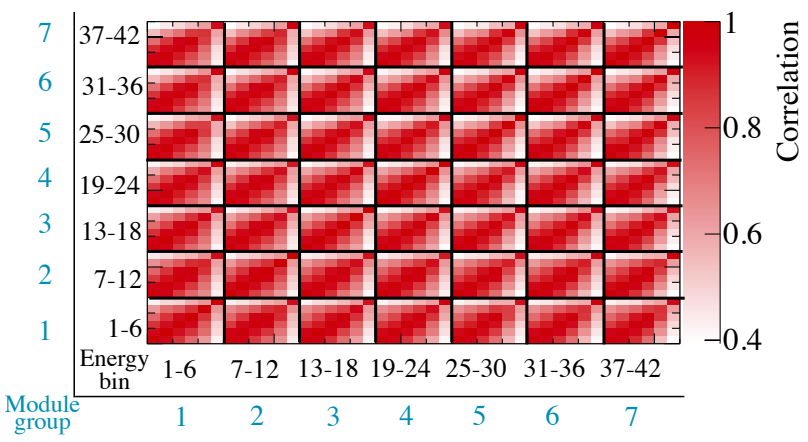

FIG. 14. Correlation matrix between module groups for the flux error. The energy binning used in this matrix is same as the "lobal bin" defined in Table V.

\section{B. Neutrino interaction model uncertainties}

Any systematic error in the CC interaction model would, by definition, alter the $\mathrm{CC}$ inclusive cross section itself. This is not the case for NC interaction model uncertainties, and so the systematic errors associated with these two processes are evaluated separately.

\section{Systematics uncertainty on NC interactions}

The uncertainty in the number of NC interactions in each bin is expressed by the normalization parameter:

$$
f_{j g}^{n c} \equiv \frac{N_{j g}^{\prime n c}}{N_{j g}^{n c}},
$$

where $N_{j g}^{n c}$ is the predicted number of NC events for the $j^{t h}$ topology and the $g^{t h}$ module group. $N_{j g}^{\prime n c}$ is the predicted number of events in the same bin but for the case where one of the $\mathrm{NC}$ systematic parameters has been changed by $1 \sigma$. The number of events is altered not only by the change in the cross section but also by changes in the event detection efficiency. The same normalization parameter, $f_{j g}^{n c}$, is used for all module groups, so the number of predicted events, $N_{j g}^{n c}$, becomes:

$$
N_{j g}^{n c} \rightarrow f_{j}^{n c} \cdot N_{j g}^{n c} \quad\left(f_{j}^{n c}=f_{j g}^{n c}\right) .
$$

$f_{j}^{n c}$ is estimated by combining NC events from all module groups. The fractional covariance for the topology bins is then calculated by varying each $\mathrm{NC}$ systematic parameter by $\pm 1 \sigma$.

$$
\begin{aligned}
V_{i j}^{n c}= & \frac{1}{2}\left[\left(\frac{N_{i}^{n c}-N_{i}^{n c,+1 \sigma}}{N_{i}^{n c}} \cdot \frac{N_{j}^{n c}-N_{j}^{n c,+1 \sigma}}{N_{j}^{n c}}\right)\right. \\
& \left.+\left(\frac{N_{i}^{n c}-N_{i}^{n c,-1 \sigma}}{N_{i}^{n c}} \cdot \frac{N_{j}^{n c}-N_{j}^{n c,-1 \sigma}}{N_{j}^{n c}}\right)\right],
\end{aligned}
$$

where $N_{i(j)}^{n c}$ is obtained by summing over all module groups:

$$
N_{i}^{n c}=\sum_{g} N_{i g}^{n c} .
$$

We found that the total NC error is fully correlated between all of the "NonDS-escaping" bins. Therefore, the 7 "NonDS-escaping" bins are merged into a single bin. Figure 15 shows the correlation matrix for the NC interactions uncertainty, demonstrating that the event topology bins are almost fully correlated with each other. This correlation comes mainly from the NC normalization error (see Table II).

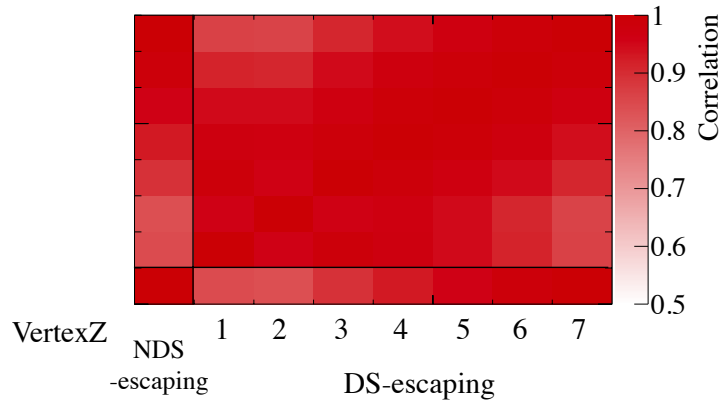

FIG. 15. Correlation matrix for the errors coming from $\mathrm{NC}$ interaction uncertainties. The binning on the y-axis is identical to that on the $\mathrm{x}$-axis.

The total normalization error on the number of $\mathrm{NC}$ events is $27-30 \%$, which is dominated by the NC normalization error, which is shown in Fig. 16. This gives a maximum error size of $5 \%$ on the total $(\mathrm{CC}+\mathrm{NC})$ number of events.

\section{Systematic uncertainty on $C C$ interactions}

Varying the $\mathrm{CC}$ interaction parameters results in a change in both the neutrino cross section and the de- 


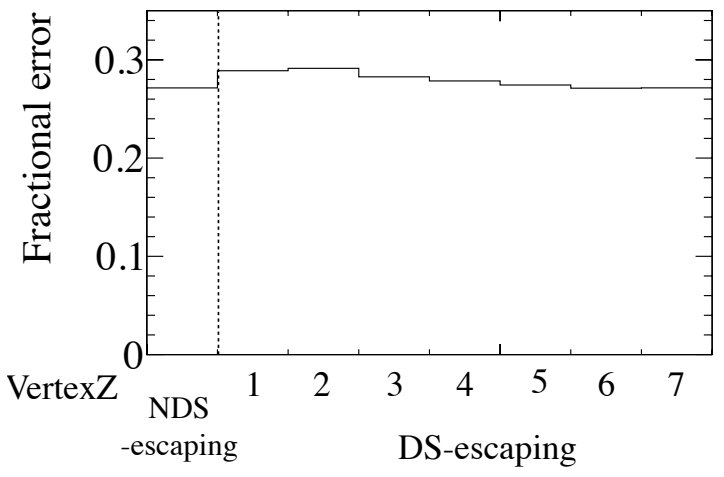

FIG. 16. Fractional error from NC interaction uncertainties on the number of NC events.

tection efficiency. In this analysis, for the systematic uncertainty on CC interactions, only the latter change is taken into account, since the result of the analysis will be the cross section itself. A $1 \sigma$ variation is applied to a $\mathrm{CC}$ interaction parameter and the new selection efficiency, $\epsilon_{i j}^{\prime c c}$ is calculated. The change in the detection efficiency is then given by the ratio of the new efficiency to the nominal one:

$$
w_{i j}=\frac{\epsilon_{i j}^{\prime c c}}{\epsilon_{i j}^{c c}},
$$

where the indices $i$ and $j$ denote the $i^{\text {th }}$ energy bin and the $j^{\text {th }}$ topology bin, respectively. The predicted number of CC events is then modified using $w_{i j}$ :

$$
N_{j g}^{\prime c c}=\sum_{i} \phi_{i g} \cdot \sigma_{i}^{c c} \cdot\left(w_{i j} \epsilon_{i j}^{c c}\right) \cdot T,
$$

A fractional covariance between topology bins is then calculated for each CC interaction parameter using the modified $\left(N^{\prime c c}\right)$ and nominal number of CC events. This is performed in the same way as for the $\mathrm{NC}$ interaction uncertainty, described earlier. The total covariance matrix is computed by summing up the individual matrix from each CC interaction parameter. The obtained correlation matrix and fractional error between the event topology bins are shown in Figs. 17 and 18.

\section{Systematic uncertainty on FSI}

For the pion FSI parameters, uncertainties on the absorption, charged exchange, quasi-elastic, and inelastic scattering cross sections of the pion are taken into account. These systematic errors are treated in a different to the previous interaction uncertainties because there are correlations between them. The INGRID data is fitted with $N^{\prime c c}$, obtained by changing each FSI parameter by $1 \sigma$, and the difference between the fitted and the nominal result is taken as the systematic error. The effect of each FSI parameter on the measured cross section was found to be negligible, except for the pion absorption

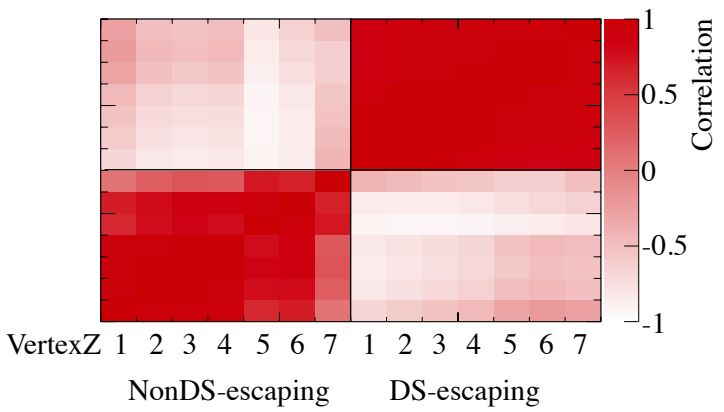

FIG. 17. Correlation matrix for the errors coming from $\mathrm{CC}$ interaction uncertainties. The binning on the y-axis is identical to that on the $\mathrm{x}$-axis.

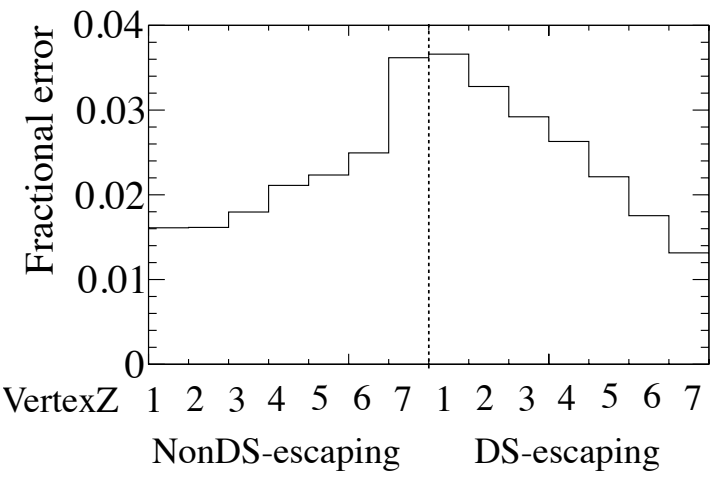

FIG. 18. Fractional error from CC interaction uncertainties.

uncertainty, which was then added in quadrature to the final result.

\section{Detector response uncertainties}

As described in Sec.IVE, each error source is categorized according to whether it produces correlation among topology bins or not. For the uncorrelated error sources, the iron mass and pileup correction, the individual systematic errors are summed quadratically, and the total inserted into the denominator of the $\chi^{2}$ statistical term, $\left(\sigma_{N_{j g}^{\text {det }}}\right.$, in Eq. $\left.(5)\right)$.

For the correlated error sources, the size of the error does not to vary between each module, so a covariance matrix is constructed from the topology bins using the average change over all modules. Namely, the errors are assumed to be fully correlated between module groups. For uncertainties from the event selection, the systematic error is evaluated by varying each selection threshold by $1 \sigma$, and the resultant fractional variation in the number of selected events $(\equiv \Delta N / N)$ computed for data and MC. The difference in $\Delta N / N$ between data and MC is then taken as the systematic error, calculated as:

$$
\Delta_{j}=\left(\frac{\Delta N_{o b s}}{N_{o b s}}\right)_{j}-\left(\frac{\Delta N_{e x p}}{N_{e x p}}\right)_{j},
$$


where the index $j$ denotes the $j^{\text {th }}$ topology bin. If both a $+1 \sigma$ and $-1 \sigma$ variation are applied to the event selection then the following covariance is calculated:

$$
V_{i j}=\frac{1}{2}\left\{\left(\Delta_{i} \cdot \Delta_{j}\right)_{+1 \sigma}+\left(\Delta_{i} \cdot \Delta_{j}\right)_{-1 \sigma}\right\} .
$$

If only a $+1 \sigma$ change can be applied the the covariance becomes:

$$
V_{i j}=\Delta_{i} \cdot \Delta_{j} .
$$

The statistical error of $\Delta_{i}$ is also calculated and added to Eq. (17) (or Eq. (18)). Finally, the total covariance is calculated by summing each individual covariance. Figures 19 and 20 show the correlation matrix obtained and the size of the fractional error for each event topology bin.

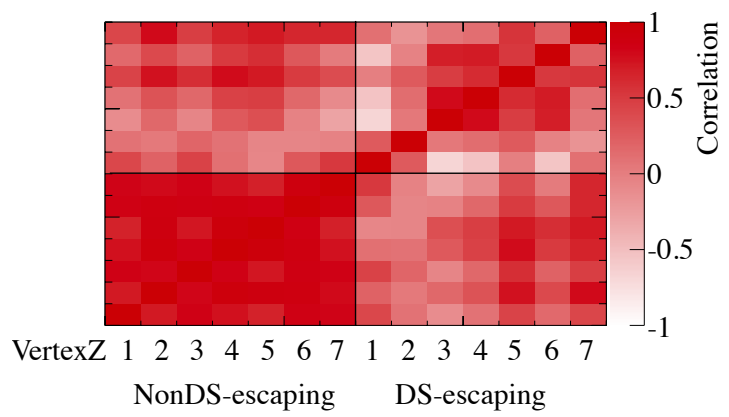

FIG. 19. Correlation matrix from the uncertainties in the detector response. The binning on the y-axis is identical to that on the $\mathrm{x}$-axis.

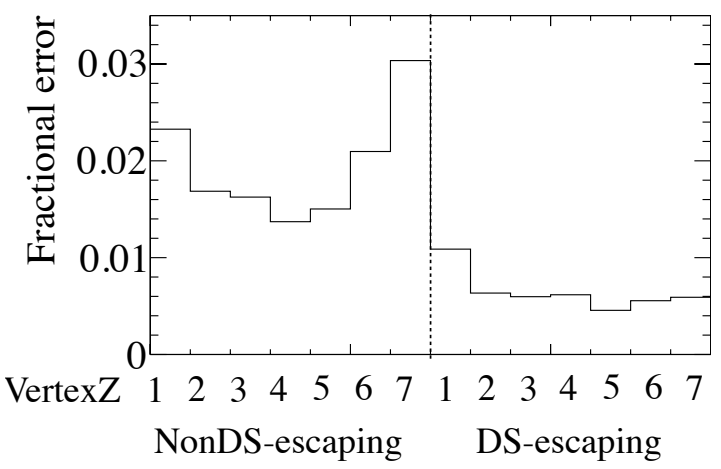

FIG. 20. Fractional error for the topology bins coming from uncertainties in the detector response.

TABLE VI. Systematic error size on topology bins due to uncertainties in the detector response.

\begin{tabular}{cc}
\hline \hline Error type & Error size (at maximum) \\
\hline Correlated error & $3 \%$ \\
Uncorrelated error & $2 \%$ \\
\hline \hline
\end{tabular}

Table VI summarizes the size of the detector systematic error for each error type.

\section{Uncertainty in pion multiplicities and secondary interactions}

Uncertainties associated with pion multiplicities and pion secondary interactions (SI) are treated in a different way to the systematics described above. These uncertainties are evaluated by comparing the underlying model with external data. Any observed difference is used to correct the nominal MC sample, and then the $\chi^{2}$ fit is performed using this "corrected" MC. The differences in the fitted values between the nominal and corrected MC are then taken as the systematic error on the final result.

\section{Pion multiplicity:}

In this analysis, the number of events is determined from the number of reconstructed vertices, which are sometimes missed due to the pile-up of tracks from multiple neutrino interactions. Events with large numbers of tracks usually contain pions, therefore the uncertainty associated with the pion multiplicity in these events needs to be considered. This uncertainty is estimated by following the method described in Ref. [38]. The probability of an event having a pion multiplicity of $n$ is expressed as:

$$
\begin{aligned}
P(z ; A, B, c) & =\frac{1}{\langle n\rangle} \frac{2 e^{-c} c^{c z+1}}{\Gamma(c z+1)} \\
\langle n\rangle & =A+B \log W^{2}
\end{aligned}
$$

where $z=n /\langle n\rangle ;\langle n\rangle$ is the mean pion multiplicity and can be expressed by an approximate formula as in Eq. (20). The $W$ is the hadronic invariant mass, and is expressed as:

$W^{2}=\left(E_{\nu}+E_{N}-E_{\mu}\right)^{2}-\left(\vec{p}_{\nu}+\vec{p}_{N}-\vec{p}_{\mu}\right)^{2}$

with $E_{\nu_{\mu}}\left(\vec{p}_{\nu_{\mu}}\right)$ and $E_{\mu}\left(\vec{p}_{\mu}\right)$ denoting the energy (3momentum) of the $\nu_{\mu}$ and $\mu$ respectively; $\vec{p}_{N}$ denoting the Fermi momentum of the nucleon and $E_{N}$ the nucleon energy. $A, B$, and $c$ are derived by fitting two external data sets [39, 40] with Eq. (19). These fitted parameters are compared with those used in NEUT, and the differences assigned as the systematic uncertainty. The parameters are used to produce a corrected MC sample which is input to the $\chi^{2}$ fit. The differences in the fitted values coming from the corrected and the nominal MC are taken as the systematic error on the final result due to pion multiplicity uncertainties.

\section{Pion SI:}

Hadrons produced in neutrino interactions can also interact whilst traveling through the detector, a process known as "secondary interaction (SI)". In the INGRID simulation the pion SI processes are controlled by GEANT4. In order to evaluate the uncertainty in the pion SI model, the following interaction modes are considered: 
TABLE VII. Summary of pion-nucleus scattering data used to evaluate the pion SI uncertainty. The reactive cross section is defined as the sum of all the inelastic cross sections.

\begin{tabular}{|c|c|c|c|c|}
\hline & Hadrons & Targets & 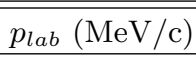 & Interaction type \\
\hline K. Nakai et al. [33] & $\overline{\pi^{+} / \pi^{-}}$ & $\mathrm{Al}, \mathrm{Ti}, \mathrm{Cu}, \mathrm{Sn}, \mathrm{Au}$ & $83-395$ & ABS \\
\hline D. Ashery et al. [34] & $\pi^{+} / \pi^{-}$ & $\mathrm{Li}, \mathrm{C}, \mathrm{O}, \mathrm{Al}, \mathrm{Fe}, \mathrm{Nb}, \mathrm{Bi}$ & $175-432$ & Reactive, Elastic, QEL, ABS, SCX \\
\hline M.K. Jones et al. [35] & $\pi^{+}$ & $\mathrm{C}, \mathrm{Ni}, \mathrm{Zr}, \mathrm{Sn}, \mathrm{Pb}$ & $363-624$ & QEL, ABS, SCX \\
\hline G.J. Gelderloos et al. [36] & $\pi^{-}$ & $\mathrm{Li}, \mathrm{C}, \mathrm{Al}, \mathrm{S}, \mathrm{Ca}, \mathrm{Cu}, \mathrm{Zr}, \mathrm{Sn}, \mathrm{Pb}$ & $479-616$ & Reactive \\
\hline B.W. Allardyce et al. [37] & $\pi^{+} / \pi^{-}$ & $\mathrm{C}, \mathrm{Al}, \mathrm{Ca}, \mathrm{Ni}, \mathrm{Sn}, \mathrm{Ho}, \mathrm{Pb}$ & $710-2000$ & Reactive \\
\hline
\end{tabular}

Quasi-elastic scattering (QEL):

The final state pion is the same type as the incoming pion.

\section{Absorption (ABS):}

The incident pion is absorbed by the nucleus, resulting in there being no pions in the final state.

\section{Single charge exchange (SCX):}

A charged pion interacts such that there is only one $\pi^{0}$ and no other pions in the final state.

The existing experimental data used to evaluate this uncertainty is summarized in Table VII. In the table, the reactive cross section is defined as $\sigma_{\text {total }}-\sigma_{\text {elastic }}$, where $\sigma_{\text {total }}$ is the total cross section and $\sigma_{\text {elastic }}$ is the elastic cross section. As seen in the table, D. Ashery et al. provides various cross sections across a range of pion momenta and target nuclei, including iron. The other data do not include measurements on iron. For these data, an $A$-dependent scaling is applied in order to extract the cross section on iron. To evaluate the systematic uncertainty coming from pion SI, we first tune the pion cross section in the momentum region covered by the data in Table VII. Second, for the lower energy region not covered by data, the $\operatorname{ABS} \pi^{+}\left(\pi^{-}\right)$ cross section $<20(30) \mathrm{MeV}$ is kept constant, motivated by the microscopic calculation from [41]. The QEL cross section is extrapolated to 0 at $0 \mathrm{MeV}$. For the higher energy region, each of the cross sections above is tuned on the basis that the size of the reactive cross section is conserved, since the cross sections predicted by GEANT4 are in agreement with the experimental data in this energy region. This study gives 4 corrected MC samples in total, each of which is then used to fit the T2K data. Finally, the size of the systematic error on the $f_{k}$ parameters due to the uncertainty on pion FSI is calculated as follows:

$$
\Delta f_{k}=\sqrt{\frac{1}{4} \sum_{i=1}^{4}\left(f_{k}^{n o m}-f_{k}^{i}\right)^{2}},
$$

where the index $i$ denotes the $i^{t h}$ corrected MC sample and $f_{k}$ is the fitted normalization parameter for the $k^{t h}$ energy bin.

\section{RESULT}

In this section, we present the result of this $\nu_{\mu}$ inclusive $\mathrm{CC}$ cross-section measurement. Section VI A shows the data set used in this analysis, whilst Secs. VIB and VIC describe the output from the $\chi^{2}$ fit and give a summary of this result.

\section{A. Data set}

Figure 21 shows the observed and predicted topology distributions in all module groups for the data set used in this analysis, corresponding to $6.27 \times 10^{20}$ p.o.t. The number of observed events for the NonDS-escaping topology is $3-10 \%$ smaller than expected.

\section{B. Cross section fit}

The topology distributions after the data fit are shown in Fig. 22. As seen in the figure, the predicted topology distributions with the fitted cross-section normalization parameters applied agree well with the observed distributions.

Table VIII shows the cross-section normalization parameters, $f_{i}=1+\Delta f_{i}(\mathrm{i}=0-4)$, obtained by fitting the INGRID data, where the $\Delta f_{i}$ 's are those from Eq. (6).

The fitted values for the flux, detector, CC interaction, and $\mathrm{NC}$ interaction uncertainty parameters are shown in Fig. 23. A large deviation from 0 is seen for all the NC interaction uncertainty parameters. As described in Sec. VIA, the prediction overestimates the number of NonDS-escaping events by $3-10 \%$.

The fitter preferentially reduces the number of $\mathrm{NC}$ events to match the predicted topology distribution to the observed one. Since the NC interaction uncertainty parameters are almost fully correlated among topologies, as shown in Fig. 15, all the parameters move toward negative values. There are jumps seen in the $\mathrm{CC}$ interaction and detector uncertainty parameters. Both of the jumps 

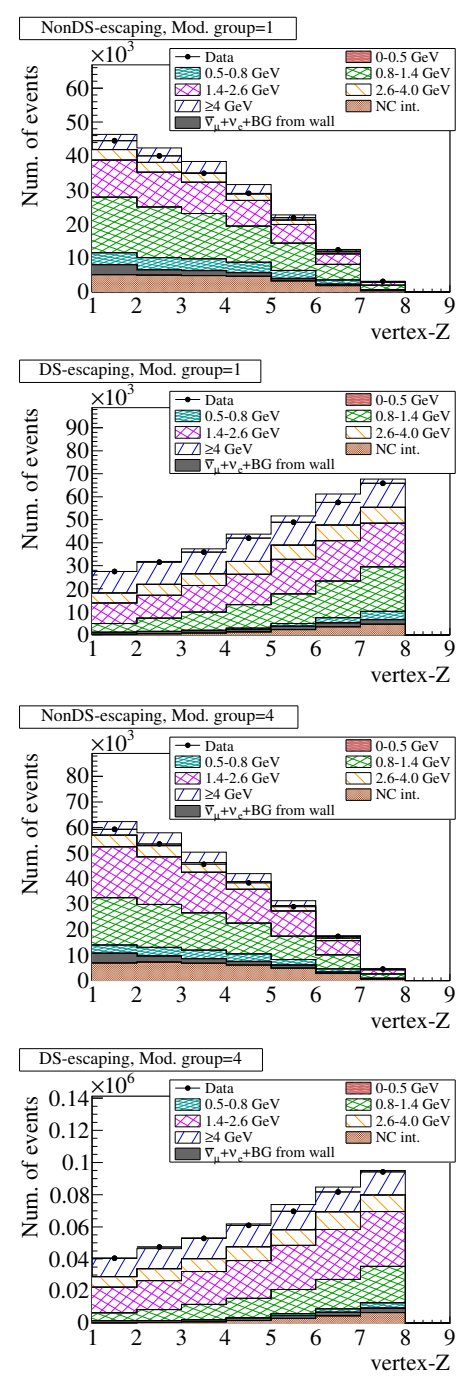
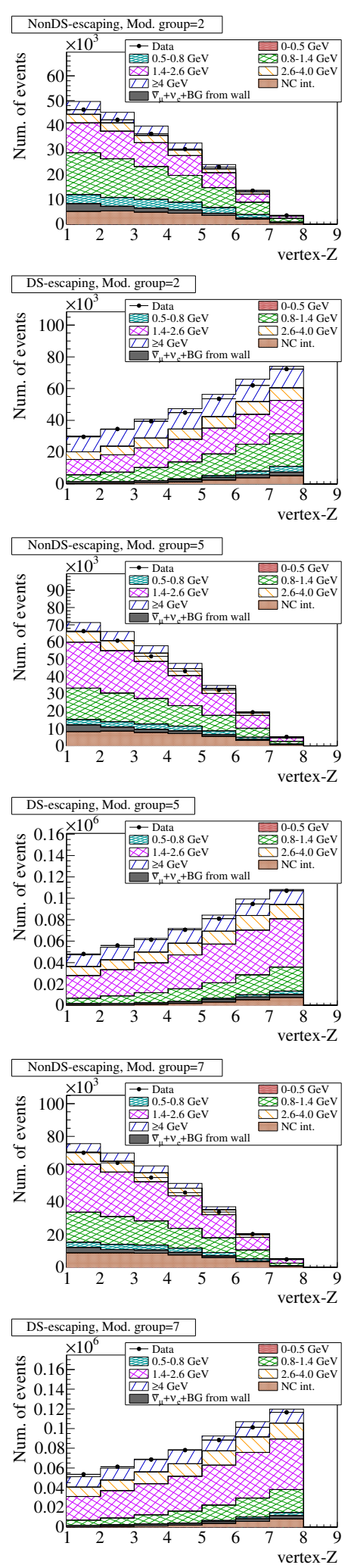
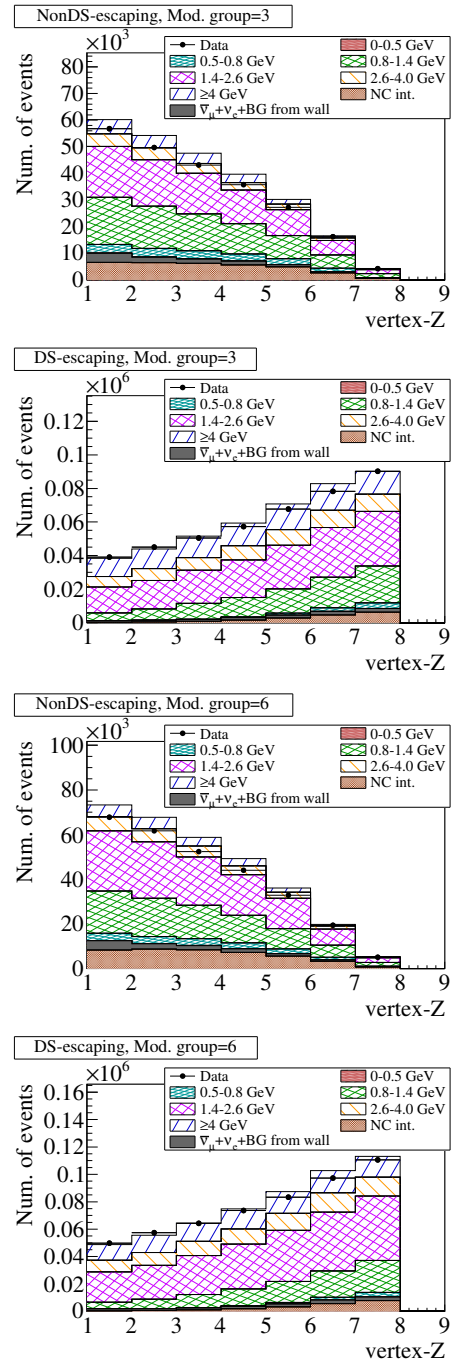

FIG. 21. Topology distribution for NonDS-escaping (top) and DS-escaping (bottom) for all module groups. The predicted events, before the fit, are categorized as CC events, NC events, and either intrinsic beam $\bar{\nu}_{\mu}+\nu_{e}$ backgrounds or backgrounds from the wall, and shown as a stacked histogram. The CC events are subdivided into 6 true neutrino energy regions. 

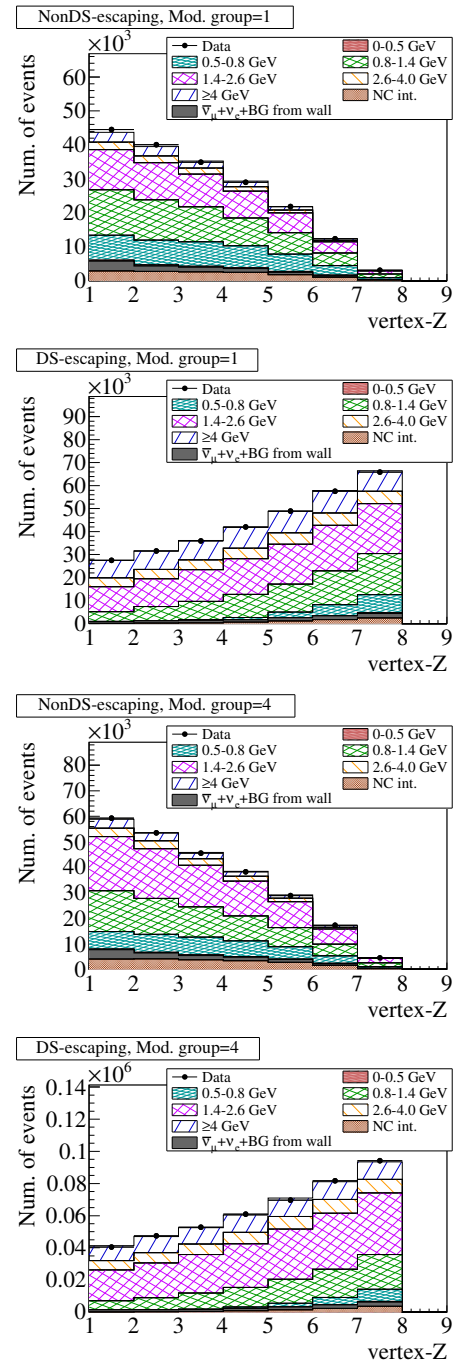
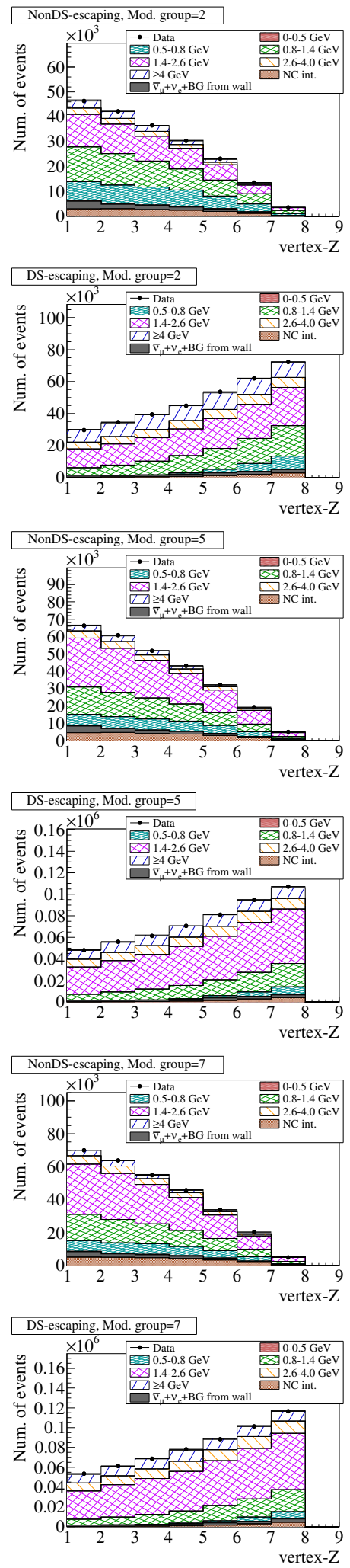
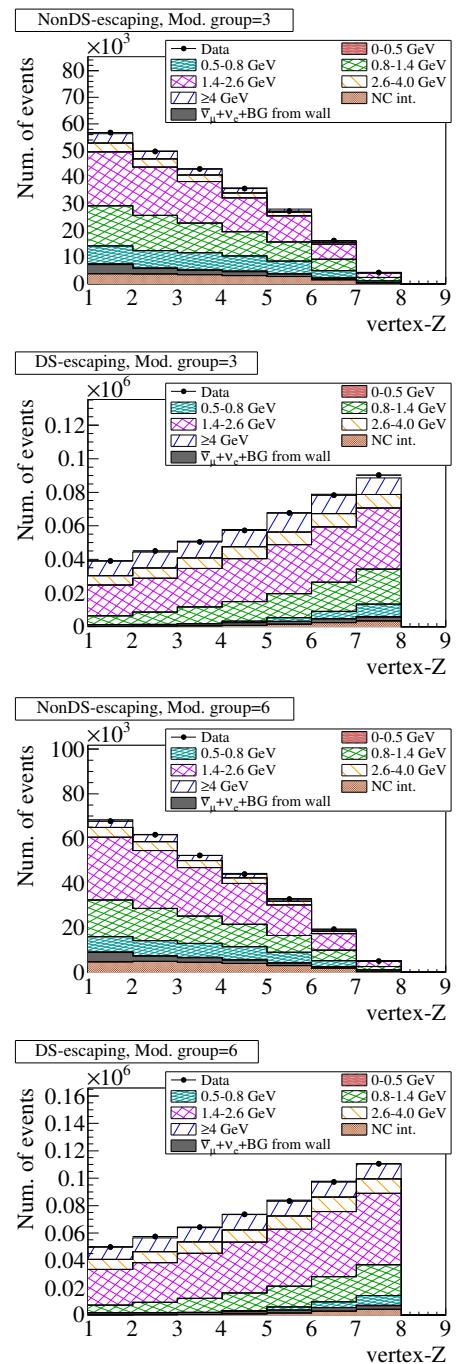

FIG. 22. Topology distribution for NonDS-escaping (top) and DS-escaping (bottom) events for all module groups after the data fit. The predicted events are categorized as CC events, $\mathrm{NC}$ events, and either intrinsic beam $\bar{\nu}_{\mu}+\nu_{e}$ backgrounds or backgrounds from the wall, and shown as a stacked histogram. The CC events are subdivided into 6 true neutrino energy regions. 
TABLE VIII. Fitted values of the cross-section normalization parameters, $f_{i}=1+\Delta f_{i}$.

\begin{tabular}{c|c}
\hline \hline Fit parameter & Fit result \\
\hline$f_{0}(0.5 \mathrm{GeV})$ & $3.560 \pm 0.508$ \\
$f_{1}(0.8 \mathrm{GeV})$ & $0.637 \pm 0.180$ \\
$f_{2}(1.4 \mathrm{GeV})$ & $1.324 \pm 0.181$ \\
$f_{3}(2.6 \mathrm{GeV})$ & $0.800 \pm 0.211$ \\
$f_{4}(4.0 \mathrm{GeV})$ & $0.712 \pm 0.120$ \\
\hline$\chi^{2}$ & 155.4 \\
\hline \hline
\end{tabular}
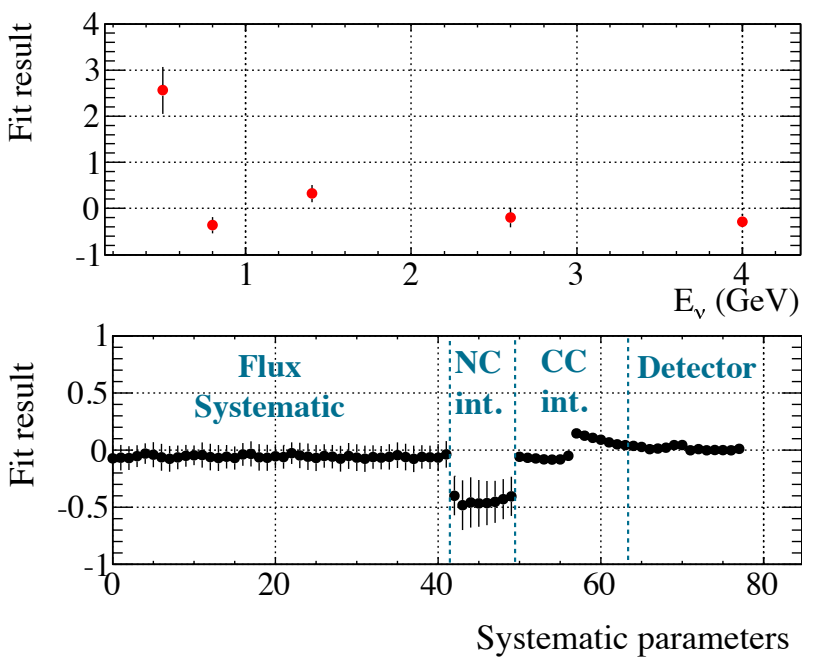

FIG. 23. Fitted values of the cross-section normalization (top) and systematic (bottom) parameters.

appear at the boundary between parameters for NonDSescaping and DS-escaping events.

In order to derive the normalization factor for the cross section, we take the average of the neighboring fitted $f_{i}$ parameters. The obtained cross-section normalizations are:

$$
\begin{aligned}
f(1.1 \mathrm{GeV}) & =\frac{f_{1}+f_{2}}{2} \\
& =0.980 \pm 0.115 \\
f(2.0 \mathrm{GeV}) & =\frac{f_{2}+f_{3}}{2} \\
& =1.062 \pm 0.123, \\
f(3.3 \mathrm{GeV}) & =\frac{f_{3}+f_{4}}{2} \\
& =0.756 \pm 0.136,
\end{aligned}
$$

Table IX summarizes the uncertainty on the fitted cross-section normalization parameters, broken down by error source. The errors on the combined normalization parameters are summarized in Table X. The largest systematic error source is the flux uncertainty, which gives a $8-9 \%$ error on the cross-section normalization. The cross-section normalization at $2.0 \mathrm{GeV}$ is less affected by most of the systematic uncertainties than the other normalizations, as shown in Table $\mathrm{X}$. The reason for this is as follows. In Fig. 13, one can see that the PDF is well differentiated for both module group and event topology at $E_{\nu}=1.4-2.6 \mathrm{GeV}\left(f_{2}-f_{3}\right)$ and $E_{\nu}=2.6-4.0 \mathrm{GeV}\left(f_{3}-f_{4}\right)$, while poor differentiation is seen for $E_{\nu} \geq 4.0 \mathrm{GeV}\left(f_{4}\right)$, which results in weak correlation with the other crosssection normalizations. Thus, sensitivity to the crosssection normalization is good for $E_{\nu}=2.0 \mathrm{GeV}\left(=\frac{f_{2}+f_{3}}{2}\right)$ but limited for $E_{\nu}=3.3 \mathrm{GeV}\left(=\frac{f_{3}+f_{4}}{2}\right)$.

TABLE IX. Contribution to the uncertainty on the fitted parameters $\left(f_{0}-f_{4}\right)$ from each error source.

\begin{tabular}{lccccc}
\hline \hline Error source & $\begin{array}{c}f_{0} \\
(0.5 \mathrm{GeV})\end{array}$ & $\begin{array}{c}f_{1} \\
(0.8 \mathrm{GeV})\end{array}$ & $\begin{array}{c}f_{2} \\
(1.4 \mathrm{GeV})\end{array}$ & $\begin{array}{c}f_{3} \\
(2.6 \mathrm{GeV})\end{array}$ & $\begin{array}{c}f_{4} \\
(4.0 \mathrm{GeV})\end{array}$ \\
\hline Statistical error & $18.7 \%$ & $6.0 \%$ & $4.5 \%$ & $4.8 \%$ & $1.4 \%$ \\
Flux + Stat. & $26.0 \%$ & $7.9 \%$ & $12.8 \%$ & $14.5 \%$ & $9.3 \%$ \\
Detector + Stat. & $33.8 \%$ & $10.0 \%$ & $7.2 \%$ & $7.0 \%$ & $3.0 \%$ \\
Interaction (cc) + Stat. & $30.6 \%$ & $9.3 \%$ & $6.8 \%$ & $7.2 \%$ & $3.8 \%$ \\
Interaction (nc) + Stat. & $22.6 \%$ & $6.6 \%$ & $6.4 \%$ & $5.8 \%$ & $2.0 \%$ \\
\hline pion FSI & $+0.4 \%$ & $+0.3 \%$ & $+2.0 \%$ & $+4.0 \%$ & $+3.5 \%$ \\
pion multiplicity & $-0.2 \%$ & $-0.8 \%$ & $-3.3 \%$ & $-3.6 \%$ & $-5.2 \%$ \\
pion SI & $2.6 \%$ & $8.8 \%$ & $0.7 \%$ & $12.4 \%$ & $9.4 \%$ \\
\hline \hline
\end{tabular}

TABLE X. Contribution to the uncertainty on the crosssection normalization at $1.1,2.0$, and $3.0 \mathrm{GeV}$ from each error source.

\begin{tabular}{lccc}
\hline \hline Error source & $1.1 \mathrm{GeV}$ & $2.0 \mathrm{GeV}$ & $3.3 \mathrm{GeV}$ \\
\hline Statistical error & $2.0 \%$ & $0.6 \%$ & $2.3 \%$ \\
Flux + Stat. & $7.6 \%$ & $9.0 \%$ & $8.4 \%$ \\
Detector + Stat. & $4.3 \%$ & $0.9 \%$ & $3.9 \%$ \\
Interaction (cc) + Stat. & $3.7 \%$ & $0.8 \%$ & $4.8 \%$ \\
Interaction (nc) + Stat. & $2.4 \%$ & $0.9 \%$ & $3.2 \%$ \\
\hline pion FSI & $+1.0 \%$ & $0.5 \%$ & $+3.7 \%$ \\
pion multiplicity & $3.3 \%$ & $5.1 \%$ & $2.1 \%$ \\
pion SI & $5.6 \%$ & $2.0 \%$ & $6.9 \%$ \\
\hline \hline
\end{tabular}

Figures 24 and 25 show the error and correlation matrices for the 5 fitted parameters $\left(\Delta f_{0}-\Delta f_{4}\right)$ and the crosssection normalizations at $1.1,2.0$, and $3.3 \mathrm{GeV}$, respectively.

\section{Summary}

In the previous section, five individual fitting parameters were extracted with the least $\chi^{2}$ method, and used to calculate the following measured cross sections at en- 


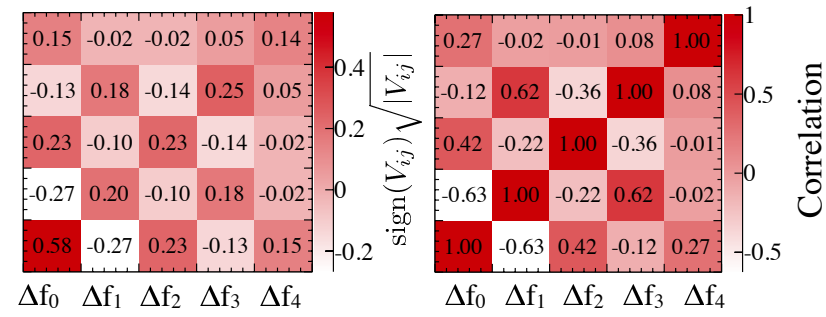

FIG. 24. Error (left) and correlation (right) matrices for the 5 fitted parameters. In both of the matrices, the binning on the $\mathrm{y}$-axis is identical to that on the $\mathrm{x}$-axis.

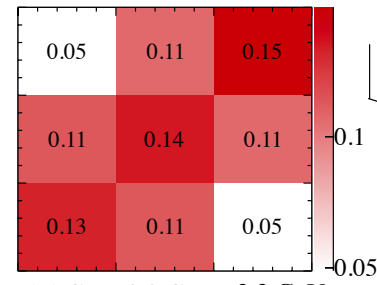

$1.1 \mathrm{GeV} 2.0 \mathrm{GeV} 3.3 \mathrm{GeV}$

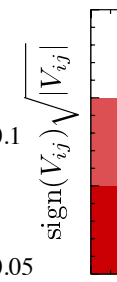

$1.1 \mathrm{GeV} 2.0 \mathrm{GeV} 3.3 \mathrm{GeV}$
FIG. 25. Error (left) and correlation (right) matrices for the cross-section normalization at $1.1,2.0$, and $3.3 \mathrm{GeV}$. In both of the matrices, the binning on the $y$-axis is identical to that on the x-axis.

ergies of 1.1, 2.0, and $3.3 \mathrm{GeV}$ :

$$
\begin{aligned}
& \sigma^{c c}(1.1 \mathrm{GeV})=1.10 \pm 0.15\left(10^{-38} \mathrm{~cm}^{2} / \text { nucleon }\right) \\
& \sigma^{c c}(2.0 \mathrm{GeV})=2.07 \pm 0.27\left(10^{-38} \mathrm{~cm}^{2} / \text { nucleon }\right) \\
& \sigma^{c c}(3.3 \mathrm{GeV})=2.29 \pm 0.45\left(10^{-38} \mathrm{~cm}^{2} / \text { nucleon }\right)
\end{aligned}
$$

Figure 26 shows these results compared to other measurements $[1,2]$ and the neutrino event generators, NEUT (ver.5.1.4.2) and GENIE (ver.2.8.0). These measurements are consistent with the energy dependent cross section measured by the MINOS near detector and the previous, flux-averaged, cross section measured by INGRID, which used a subset of the data included in this analysis.

This analysis utilizes the different off-axis angle technique together with the final state kinematics of the outgoing lepton to enhance sensitivity to the neutrino energy. Using final state lepton kinematics in this analysis makes the result sensitive to uncertainties in the neutrino interaction model, which increases the uncertainty on the final measurement. As a result, the errors achieved are not small enough to distinguish between the neutrino models used in the different event generators. Nevertheless, the result seems to prefer NEUT for $E_{\nu} \leq 2 \mathrm{GeV}$ and agrees with the MINOS data point and GENIE at $E_{\nu}=3.3 \mathrm{GeV}$. Further reduction of the systematic error size would help in differentiating the neutrino models at the higher energy transition. This reduction could be made by utilizing neutrino beams covering a wider range of off-axis angles, which would provide a "model independent" way to infer the neutrino energy. This analysis demonstrates the feasibility of using different off-axis samples from the same neutrino beam to measure the energy dependence of neutrino interactions, which will provide useful information for future neutrino oscillation analyses.

\section{CONCLUSION}

In this paper, we have reported the measurement of the energy dependent inclusive $\nu_{\mu}$ charged current cross section on iron using the T2K INGRID detector and the $\mathrm{T} 2 \mathrm{~K}$ neutrino beam. The unique variation in the neutrino flux across the INGRID modules, along with event kinematic information, was used to produce event samples sensitive to neutrinos with energies from 1-3 GeV. These were used to extract the inclusive CC muon neutrino interaction cross section on iron at 1.1, 2.0 and 3.3 $\mathrm{GeV}$, using data corresponding to $6.27 \times 10^{20}$ p.o.t. This result is consistent with the predictions of the NEUT and GENIE neutrino interaction generators. This is the first measurement to use the off-axis effect to measure neutrino cross sections as a function of energy and demonstrates the potential of this technique to provide useful information for future neutrino experiments.

\section{ACKNOWLEDGMENTS}

We thank the J-PARC staff for superb accelerator performance and the CERN NA61 collaboration for providing valuable particle production data. We acknowledge the support of MEXT, Japan; NSERC (grant SAPPJ-2014-00031), NRC and CFI, Canada; CEA and CNRS/IN2P3, France; DFG, Germany; INFN, Italy; National Science Centre (NCN), Poland; RSF, RFBR and MES, Russia; MINECO and ERDF funds, Spain; SNSF and SERI, Switzerland; STFC, UK; and DOE, USA. We also thank CERN for the UA1/NOMAD magnet, DESY for the HERA-B magnet mover system, NII for SINET4, the WestGrid and SciNet consortia in Compute Canada, and GridPP, UK. In addition participation of individual researchers and institutions has been further supported by funds from: ERC (FP7), H2020 RISE-GA644294JENNIFER, EU; JSPS, Japan; Royal Society, UK; DOE Early Career program, USA.
[1] P. Adamson et al. Neutrino and Antineutrino Inclusive Charged-current Cross Section Measurements with the
MINOS Near Detector. Phys.Rev., D81:072002, 2010. 


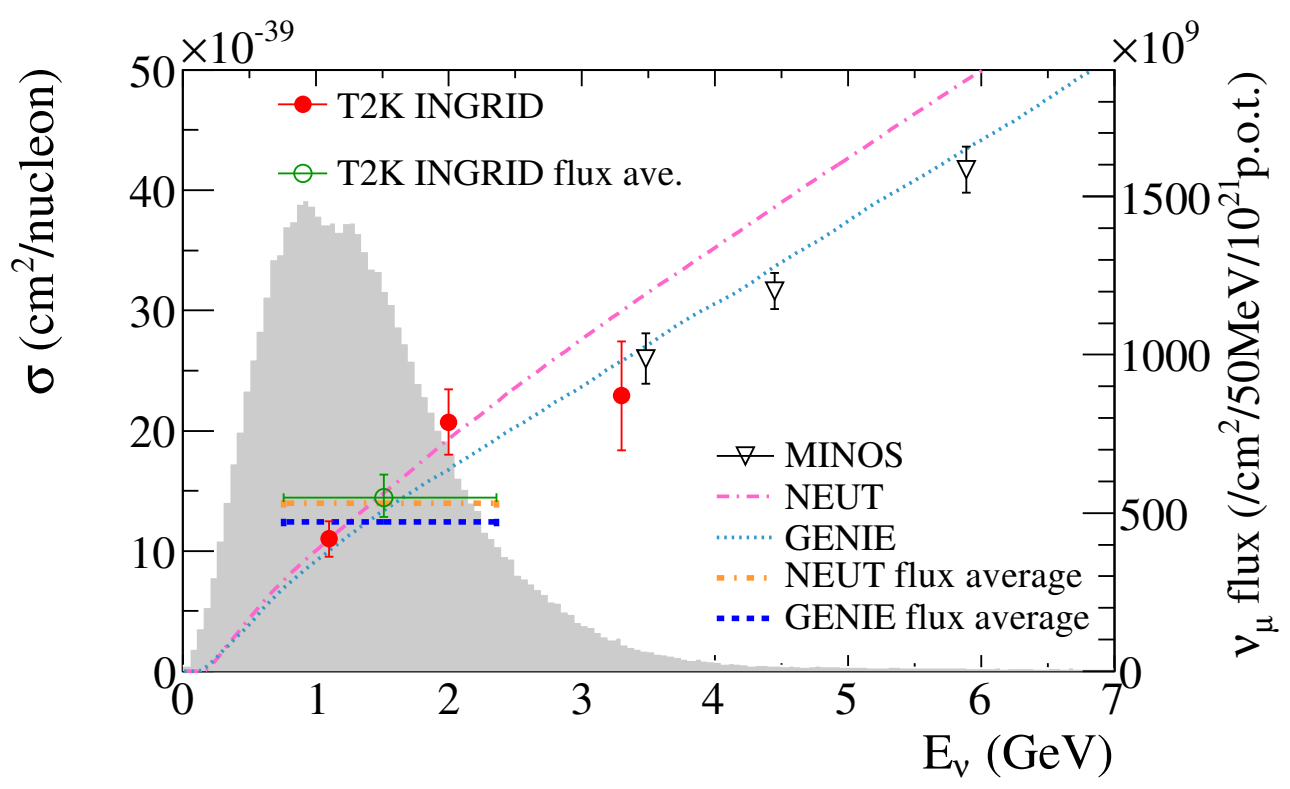

FIG. 26. Results of the $\nu_{\mu}$ CC inclusive cross section on Fe. The energy dependent cross section measured by the MINOS near detector [1] and the flux-averaged cross section from INGRID [2] are shown with the NEUT (v.5.1.4.2) and GENIE (v.2.8.0) predictions. The T2K on-axis $\nu_{\mu}$ flux is shown in gray. The T2K INGRID flux-averaged cross-section measurement and this result are consistent with one another.

[2] K. Abe et al. Measurement of the inclusive $\nu_{\mu}$ charged current cross section on iron and hydrocarbon in the T2K on-axis neutrino beam. Phys.Rev., D90(5):052010, 2014.

[3] B.G. Tice et al. Measurement of Ratios of $\nu_{\mu}$ ChargedCurrent Cross Sections on $\mathrm{C}, \mathrm{Fe}$, and $\mathrm{Pb}$ to $\mathrm{CH}$ at Neutrino Energies 2-20 GeV. Phys.Rev.Lett., 112(23):231801, 2014.

[4] K. Abe et al. The T2K Experiment. Nucl.Instrum.Meth., A659:106-135, 2011.

[5] M. Yokoyama, T. Nakaya, S. Gomi, A. Minamino, N. Nagai, et al. Application of Hamamatsu MPPC to T2K Neutrino Detectors. Nucl.Instrum.Meth., A610:128-130, 2009.

[6] M. Yokoyama, A. Minamino, S. Gomi, K. Ieki, N. Nagai, et al. Performance of Multi-Pixel Photon Counters for the T2K near detectors. Nucl.Instrum.Meth., A622:567$573,2010$.

[7] A. Vacherete et al. The front end readout system for the T2K-ND280. pages 1984-1991, 2007.

[8] K. Abe, N. Abgrall, Y. Ajima, H. Aihara, J.B. Albert, et al. Measurements of the T2K neutrino beam properties using the INGRID on-axis near detector. Nucl.Instrum.Meth., A694:211-223, 2012.

[9] K. Abe et al. T2K neutrino flux prediction. Phys.Rev., D87(1):012001, 2013.

[10] Alfredo Ferrari, Paola R. Sala, Alberto Fasso, and Johannes Ranft. FLUKA: A multi-particle transport code (Program version 2005). 2005.

[11] C. Zeitnitz and T.A. Gabriel. The GEANT-CALOR interface. 1992.

[12] Rene Brun, Federico Carminati, and Simone Giani. GEANT Detector Description and Simulation Tool. 1994.
[13] N. Abgrall et al. NA61/SHINE facility at the CERN SPS: beams and detector system. JINST, 9:P06005, 2014.

[14] T. Eichten, D. Haidt, J.B.M. Pattison, W. Venus, H.W. Wachsmuth, et al. Particle production in proton interactions in nuclei at 24-GeV/c. Nucl.Phys., B44:333-343, 1972.

[15] J.V Allaby, F.G. Binon, A.N Diddens, P. Duteil, A. Klovning, et al. High-energy particle spectra from proton interactions at $19.2-\mathrm{GeV} / \mathrm{c} .1970$.

[16] N Abgrall et al. Measurements of Cross Sections and Charged Pion Spectra in Proton-Carbon Interactions at $31 \mathrm{GeV} / \mathrm{c}$. Phys.Rev., C84:034604, 2011.

[17] N. Abgrall et al. Measurement of Production Properties of Positively Charged Kaons in Proton-Carbon Interactions at $31 \mathrm{GeV} / \mathrm{c}$. Phys.Rev., C85:035210, 2012.

[18] Yoshinari Hayato. A neutrino interaction simulation program library NEUT. Acta Phys.Polon., B40:2477-2489, 2009.

[19] C. Andreopoulos, A. Bell, D. Bhattacharya, F. Cavanna, J. Dobson, et al. The GENIE Neutrino Monte Carlo Generator. Nucl.Instrum.Meth., A614:87-104, 2010.

[20] K. Abe et al. Measurement of the inclusive charged current cross section on carbon in the near detector of the T2K experiment. Phys.Rev., D87(9):092003, 2013.

[21] K. Abe et al. Evidence of Electron Neutrino Appearance in a Muon Neutrino Beam. Phys.Rev., D88(3):032002, 2013.

[22] K. Abe et al. Measurement of Neutrino Oscillation Parameters from Muon Neutrino Disappearance with an Off-axis Beam. Phys.Rev.Lett., 111(21):211803, 2013.

[23] R.A. Smith and E.J. Moniz. NEUTRINO REACTIONS ON NUCLEAR TARGETS. Nucl.Phys., B43:605, 1972.

[24] O. Benhar, B.G. Zakharov, Nikolai N. Nikolaev, and S. Fantoni. Nuclear effects in the diffractive electropro- 
duction of s anti-s mesons. Phys.Rev.Lett., 74:3565-3568, 1995.

[25] Alexis A. Aguilar-Arevalo et al. Measurement of nu(mu) and anti-nu $(\mathrm{mu})$ induced neutral current single pi0 production cross sections on mineral oil at $\mathrm{E}(\mathrm{nu}) \mathrm{O}(1-$ GeV). Phys.Rev., D81:013005, 2010.

[26] A.A. Aguilar-Arevalo et al. Measurement of NeutrinoInduced Charged-Current Charged Pion Production Cross Sections on Mineral Oil at $\mathrm{E}_{\nu} \sim 1 \mathrm{GeV}$. Phys.Rev., D83:052007, 2011.

[27] Patrick de Perio. NEUT pion FSI. AIP Conf. Proc., 1405:223-228, 2011.

[28] S. Agostinelli et al. GEANT4: A Simulation toolkit. Nucl.Instrum.Meth., A506:250-303, 2003.

[29] Bo Andersson, G. Gustafson, and B. Nilsson-Almqvist. A Model for Low $\mathrm{p}(\mathrm{t})$ Hadronic Reactions, with Generalizations to Hadron - Nucleus and Nucleus-Nucleus Collisions. Nucl.Phys., B281:289, 1987.

[30] Bo Nilsson-Almqvist and Evert Stenlund. Interactions Between Hadrons and Nuclei: The Lund Monte Carlo, Fritiof Version 1.6. Comput.Phys.Commun., 43:387, 1987.

[31] K. Abe et al. Measurements of neutrino oscillation in appearance and disappearance channels by the T2K experiment with $6.610^{20}$ protons on target. Phys.Rev., D91(7):072010, 2015.

[32] Stephen Wolfram. Statistical Mechanics of Cellular Automata. Rev.Mod.Phys., 55:601, 1983.

[33] D. Ashery, I. Navon, G. Azuelos, H.K. Walter, H.J. Pfeiffer, et al. True Absorption and Scattering of Pions on
Nuclei. Phys.Rev., C23:2173-2185, 1981.

[34] K. Nakai, T. Kobayashi, T. Numao, T.A. Shibata, J. Chiba, et al. MEASUREMENTS OF CROSSSECTIONS FOR PION ABSORPTION BY NUCLEI. Phys.Rev.Lett., 44:1446-1449, 1980.

[35] M.K. Jones, R.D. Ransome, V.R. Cupps, R.W. Fergerson, C.L. Morris, et al. Pion absorption above the Delta (1232) resonance. Phys.Rev., C48:2800-2817, 1993.

[36] C. J. Gelderloos, J. T. Brack, M. D. Holcomb, M. V. Keilman, D. J. Mercer, et al. Reaction and total crosssections for $400-\mathrm{MeV} 500-\mathrm{MeV}$ pi- on nuclei. Phys.Rev., C62:024612, 2000.

[37] B.W. Allardyce, C.J. Batty, D.J. Baugh, E. Friedman, G. Heymann, et al. Pion reaction cross-sections and nuclear sizes. Nucl.Phys., A209:1-51, 1973.

[38] Z. Koba, Holger Bech Nielsen, and P. Olesen. Scaling of multiplicity distributions in high-energy hadron collisions. Nucl.Phys., B40:317-334, 1972.

[39] Daria Zieminska, S. Kunori, C.Y. Chang, G.A. Snow, D. Son, et al. Charged Particle Multiplicity Distributions in neutrino $\mathrm{n}$ and neutrino $\mathrm{p}$ Charged Current Interactions. Phys.Rev., D27:47-57, 1983.

[40] P. Allen et al. Multiplicity Distributions in Neutrino Hydrogen Interactions. Nucl.Phys., B181:385, 1981.

[41] L.L. Salcedo, E. Oset, M.J. Vicente-Vacas, and C. Garcia-Recio. Computer Simulation of Inclusive Pion Nuclear Reactions. Nucl.Phys., A484:557, 1988. 